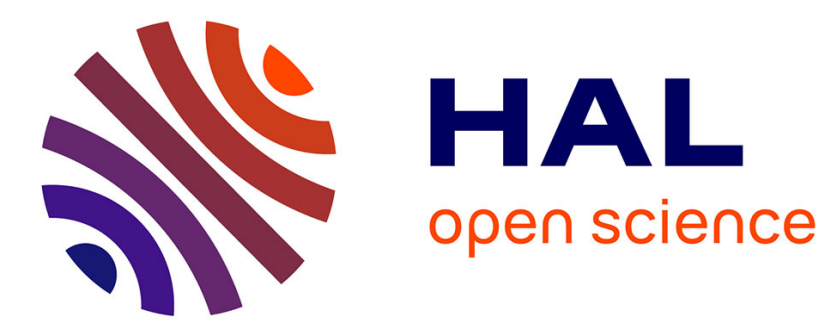

\title{
Applying Relation Algebra and RelView to Measures in aSocial Network
}

Rudolf Berghammer, Agnieszka Rusinowska, Harrie de Swart

\section{To cite this version:}

Rudolf Berghammer, Agnieszka Rusinowska, Harrie de Swart. Applying Relation Algebra and RelView to Measures in aSocial Network. 2009. halshs-00355699

\section{HAL Id: halshs-00355699 \\ https://shs.hal.science/halshs-00355699}

Submitted on 23 Jan 2009

HAL is a multi-disciplinary open access archive for the deposit and dissemination of scientific research documents, whether they are published or not. The documents may come from teaching and research institutions in France or abroad, or from public or private research centers.
L'archive ouverte pluridisciplinaire HAL, est destinée au dépôt et à la diffusion de documents scientifiques de niveau recherche, publiés ou non, émanant des établissements d'enseignement et de recherche français ou étrangers, des laboratoires publics ou privés. 


\section{Applying Relation Algebra and RelView to Measures in a Social Network}

Rudolf Berghammer, Agnieszka Rusinowska, Harrie de Swart

Janvier 2009

GATE Groupe d'Analyse et de Théorie Économique UMR 5824 du CNRS

93 chemin des Mouilles - 69130 Écully - France

B.P. $167-69131$ Écully Cedex

Tél. +33 (0)4 72866060 - Fax +33 (0)4 72866090

Messagerie électronique gate@gate.cnrs.fr

Serveur Web : www.gate.cnrs.fr 


\title{
Applying Relation Algebra and RELVIEW to Measures in a Social Network $^{\star}$
}

\author{
Rudolf Berghammer ${ }^{1}$, Agnieszka Rusinowska ${ }^{2}$ and Harrie de Swart ${ }^{3}$ \\ 1 Institut für Informatik \\ Christian-Albrechts-Universität Kiel \\ Olshausenstraße 40, 24098 Kiel, Germany \\ rub@informatik.uni-kiel.de \\ 2 GATE \\ Université Lumière Lyon 2 - CNRS \\ 93 Chemin des Mouilles - B.P. 167, 69131 Ecully Cedex, France \\ rusinowska@gate.cnrs.fr \\ 3 Department of Philosophy \\ Tilburg University \\ P.O. Box 90153, 5000 LE Tilburg, The Netherlands \\ H.C.M.deSwart@uvt.nl
}

\begin{abstract}
We present an application of relation algebra to measure players' 'strength' in a social network with influence between players. In particular, we deal with power, success, and influence of a player as measured by the Hoede-Bakker index, its generalization and modifications, and by the influence indices. We also apply relation algebra to determine followers of a coalition and the kernel of an influence function. This leads to specifications, which can be executed with the help of the BDDbased tool RELVIEW after a simple translation into the tool's programming language. As an example we consider the present Dutch parliament.
\end{abstract}

Keywords: RELVIEw, relation algebra, social network, the Hoede-Bakker index, influence index, follower, kernel

Corresponding author: Agnieszka Rusinowska

\section{Introduction}

In order to measure players' (or agents') 'strength' in a voting situation, a lot of power indices have been proposed in the course of more than fifty years (see, for instance, $[1,12,13,14,18,26,27,28$, $29,35,36,46]$, see also $[20,31,33,48]$ for an extensive analysis of most of the power indices). In the voting power literature, one may find theoretical analysis (which includes both the axiomatic and probabilistic approaches to power indices) as well as applications of power indices (especially to decision-making in the European Union and the national parliaments).

Coming from a different direction is an approach proposed in [25], where a social network with players who are to make a 'yes'-'no' decision is considered. In this framework, a decisional power (the Hoede-Bakker) index has been introduced. The essential feature of this framework is the distinction between the inclination of a player (to say 'yes' or 'no') and the final decision of the player, which can be different from his initial inclination, due to influences of others in the network. Such an influence is formally represented by an influence function. The Hoede-Bakker index has been recently studied in $[40,41,42,43]$. In [42] the authors introduce and investigate a generalization and some modifications of the Hoede-Bakker index in a social network that coincide with some standard power indices, like the Penrose measure (also called the absolute or non-normalized Banzhaf index),

\footnotetext{
^ Co-operation for this paper is supported by European Science Foundation EUROCORES Programme - LogICCC.
} 
the Coleman indices, and the König-Bräuninger index. Moreover, 'Success', 'Luck', 'Failure' and 'Decisiveness' of a player in a social network with influence between players were defined (for an analysis of success and decisiveness of a player in voting situations, see e.g. [30]). As shown in $[40,42]$, the generalized Hoede-Bakker index measures a kind of 'net Success', i.e., 'Success Failure', but if all inclination vectors are equally probable, this index coincides with the measure of 'Decisiveness'.

Although the Hoede-Bakker index has been defined in the framework of influence, in fact it does not measure the influence between players. Influence indices, influence functions, and some other concepts related to influence (like the concepts of follower of a coalition, and of kernel of an influence function) have been investigated in [21, 22, 23, 24].

Since more than two decades, relation algebra is used successfully for formal problem specification, prototyping, and algorithm development, see e.g., [11, 47, 49]. Relations are well suited for modeling and reasoning about many discrete structures (like graphs, games, Petri nets, orders and lattices) and, due to the easy and/or efficient mechanization using, for instance, Boolean matrices, successor lists or binary decision diagrams (BDDs), also for computations on them. RELVIEW (see $[4,2,9])$ is a BDD-based tool for the visualization and manipulation of relations and for prototyping and relational programming. In $[7,8,45]$ relation algebra and RELVIEw have been successfully applied to compute the set of all feasible stable governments in a coalition formation model introduced in [44]. In the present paper we like to apply the same approach, but now to calculate measures of parties' 'strength' in a social network. Determining such measures can become quite complex and requires a lot of computations. Hence, using a computer program to calculate the measures is extremely useful for real life applications of the concepts in question. To be more precise, the aim of this paper is to apply relation algebra and RELVIEW to compute the Hoede-Bakker index, its modifications, and the influence indices, and to determine the followers of a coalition and the kernel of an influence function.

The structure of the paper is the following. Section 2 introduces the framework of influence and measures of players' strength in a social network. In Section 3 we present the facts on relation algebra that are necessary to deliver the relation-algebraic specifications and algorithms of the key concepts of Section 2. In this section we also briefly describe the RELVIEW tool. How to translate the concepts of Section 2 into relation-algebraic specifications and RELVIEW-code is demonstrated in Section 4. In order to illustrate the usefulness of the approach applied in this paper, we present in Section 5 an example based on the real structure of the present Dutch Parliament. Doing so, we also refer to the concepts of dominant and central players. Finally, we present some concluding remarks in Section 6.

\section{Measures of Players' 'Strength' in a Social Network}

The framework studied in the paper is the following. We consider a social network with the set of all players (voters) denoted by $P:=\{1, \ldots, n\}$. The players make a certain acceptance-rejection decision. Each player has an inclination either to say 'yes' (denoted by 1) or 'no' (denoted by 0 ). A Boolean inclination vector, denoted by $i=\left(i_{1}, \ldots, i_{n}\right)$, indicates the inclinations of all players. All inclination vectors are assumed to be equally probable. Let $I:=\{0,1\}^{n}$ be the set of all inclination vectors. It is assumed that players may influence each other, and due to the influences in the network, the final decision of a player may be different from his original inclination. In other words, each inclination vector $i \in I$ is transformed into a decision vector $B i$, where $B: I \rightarrow I$ with $i \mapsto B i$ is the influence function, and the decision vector $B i=\left((B i)_{1}, \ldots,(B i)_{n}\right)$ indicates the final decisions made by all players. The set of all influence functions will be denoted by $\mathcal{B}$. Let $B(I)$ be the set of all decision vectors under $B$. Furthermore, we assume a group decision function $g d: B(I) \rightarrow\{0,1\}$, 
having the value 1 if the group decision is 'yes', and the value 0 if the group decision is 'no'. The set of all group decision functions will be denoted by $\mathcal{G}$.

\subsection{The Hoede-Bakker index and its modifications}

In this section we recapitulate the original Hoede-Bakker index as introduced in [25], and its generalization and modifications given in [42]. First, we introduce some notations. Given an influence function $B \in \mathcal{B}$ and a group decision function $g d \in \mathcal{G}$, we define the two subsets $I^{+}(B, g d)$ and $I^{-}(B, g d)$ of the set $I$ of all inclination vectors as follows:

$$
\begin{aligned}
& I^{+}(B, g d):=\{i \in I \mid g d(B i)=1\} \\
& I^{-}(B, g d):=\{i \in I \mid g d(B i)=0\}
\end{aligned}
$$

$I^{+}(B, g d)$ (respectively $\left.I^{-}(B, g d)\right)$ is the set of inclination vectors leading to the group decision 'yes' (respectively 'no'). Depending on the functions $B$ and $g d$, we now introduce for each player $k \in P$ four decisive sets by the following definitions:

$$
\begin{aligned}
& I_{k}^{++}(B, g d):=\left\{i \in I \mid i_{k}=1 \wedge g d(B i)=1\right\} \\
& I_{k}^{+-}(B, g d):=\left\{i \in I \mid i_{k}=1 \wedge \operatorname{gd}(B i)=0\right\} \\
& I_{k}^{-+}(B, g d):=\left\{i \in I \mid i_{k}=0 \wedge g d(B i)=1\right\} \\
& I_{k}^{--}(B, g d):=\left\{i \in I \mid i_{k}=0 \wedge g d(B i)=0\right\}
\end{aligned}
$$

$I_{k}^{++}(B, g d)$ is the set of inclination vectors with inclination 'yes' of player $k$ that lead to the group decision 'yes', $I_{k}^{+-}(B, g d)$ is the set of inclination vectors with inclination 'yes' of player $k$ that lead to the group decision 'no', $I_{k}^{-+}(B, g d)$ is the set of inclination vectors with inclination 'no' of player $k$ that lead to the group decision 'yes', and $I_{k}^{--}(B, g d)$ is the set of inclination vectors with inclination 'no' of player $k$ that lead to the group decision 'no'. When clear from the context, we will skip ' $(B, g d)$ ' in the expressions above; so, for instance, we may write $I_{k}^{+-}$instead of $I_{k}^{+-}(B, g d)$.

In order to measure the strength of the players in a voting situation of a social network, where the inclination of a player may be different from its final decision due to influences from other players, the subsequent definition has been introduced in [25] (note, that $n$ is the number of players):

Definition 2.1.1 Given $B \in \mathcal{B}$ and $g d \in \mathcal{G}$, the decisional power (the Hoede-Bakker index) of a player $k \in P$ is defined as follows:

$$
\operatorname{HB}_{k}(B, g d):=\frac{\left|I_{k}^{++}\right|-\left|I_{k}^{+-}\right|}{2^{n-1}}
$$

The definition of the original Hoede-Bakker index assumes for the used influence function $B \in \mathcal{B}$ the following axiom to be satisfied:

$$
\forall i \in I: g d(B(\bar{i}))=\neg g d(B i)
$$

In this formula, the Boolean vector $\bar{i}$ is the complement of the inclination vector $i$ and is obtained from $i$ by component-wise negation, i.e., by interchanging all 1's with 0 's, and $\neg g d(B i)$ is the negation of the Boolean value $g d(B i)$. According to this axiom, changing all inclinations leads to a change of the group decision. Hence, given player $k \in P$, when calculating the value of $\operatorname{HB}_{k}(B, g d)$, only inclination vectors with positive inclination of $k$ may be considered.

Since the definition of the decisional power under the assumption of the above axiom is quite restrictive (for instance, a game with a veto player cannot be analyzed with this condition), in [42] a generalization of the Hoede-Bakker index (1) has been proposed, in which all inclination vectors are taken into account. In the following definition, $n$ denotes again the number of players in the social network. 
Definition 2.1.2 Given $B \in \mathcal{B}$ and $g d \in \mathcal{G}$, the generalized Hoede-Bakker index of a player $k \in P$ is defined as follows:

$$
\operatorname{GHB}_{k}(B, g d):=\frac{\left|I_{k}^{++}\right|-\left|I_{k}^{-+}\right|+\left|I_{k}^{--}\right|-\left|I_{k}^{+-}\right|}{2^{n}}
$$

The value of $\mathrm{GHB}_{k}(B, g d)$ measures a kind of 'net' Success, i.e., Success - Failure, where by a successful player, given $i \in I, B \in \mathcal{B}$ and $g d \in \mathcal{G}$, we mean a player $k \in P$ whose inclination $i_{k}$ coincides with the group decision $g d(B i)$. In [42] we show that if all inclination vectors are equally probable, then the generalized Hoede-Bakker index coincides with the Penrose measure (the absolute Banzhaf index), i.e., it measures 'Decisiveness'. A decisive player is a player who is successful and changing his inclination causes a change of the group decision. In [42] we define for $n$ players also several modifications of the generalized Hoede-Bakker index that coincide with other standard power indices.

Definition 2.1.3 Given $B \in \mathcal{B}$ and $g d \in \mathcal{G}$, for each player $k \in P$ we define modifications of the generalized Hoede-Bakker index as follows:

$$
\begin{aligned}
\mathrm{M}_{1} \operatorname{GHB}_{k}(B, g d) & :=\frac{\left|I_{k}^{++}\right|-\left|I_{k}^{-+}\right|}{\left|I^{+}\right|} \\
\mathrm{M}_{2} \operatorname{GHB}_{k}(B, g d) & :=\frac{\left|I_{k}^{--}\right|-\left|I_{k}^{+-}\right|}{\left|I^{-}\right|} \\
\mathrm{M}_{3} \operatorname{GHB}_{k}(B, g d) & :=\frac{\left|I_{k}^{++}\right|+\left|I_{k}^{--}\right|}{2^{n}} \\
\mathrm{M}_{4} \operatorname{GHB}_{k}(B, g d) & :=\frac{\left|I_{k}^{++}\right|}{\left|I^{+}\right|}
\end{aligned}
$$

Furthermore, we define independently of $k$ :

$$
\operatorname{MGHB}(B, g d):=\frac{\left|I^{+}\right|}{2^{n}}
$$

It has been proved that the modifications $\mathrm{M}_{1} \mathrm{GHB}, \mathrm{M}_{2} \mathrm{GHB}, \mathrm{M}_{3} \mathrm{GHB}$ and $\mathrm{M}_{4} \mathrm{GHB}$, coincide with the Coleman's index 'to prevent action', Coleman's index 'to initiate action', the Rae index, and the König-Bräuninger index, respectively. MGHB coincides with Coleman's 'power of a collectivity to act'. Note that the modification $\mathrm{M}_{3} \mathrm{GHB}$ (the Rae index) measures Success of a player in such a social network.

\subsection{The influence indices and followers}

In [23] some concepts to measure influence between players in the presented framework have been introduced. Before formalizing these concepts, we introduce several notations for convenience. We omit braces for sets, e.g., $\{k, m\}, P \backslash\{j\}, S \cup\{j\}$ will be written as $k m, P \backslash j, S \cup j$, respectively. We also introduce for any $S \subseteq P$ such that $|S| \geq 2$ the set $I_{S}$ of all inclination vectors under which all members of $S$ have the same inclination, i.e.,

$$
I_{S}:=\left\{i \in I \mid \forall k, j \in S: i_{k}=i_{j}\right\}
$$

and define $I_{k}:=I$ for all $k \in P$. For all inclination vectors $i \in I_{S}$ we denote by $i_{S}$ the value $i_{k}$ for some player $k \in S$. Due to the definition of the set $I_{S}$, the Boolean value $i_{S} \in\{0,1\}$ does not 
depend on the choice of $k$. Based on these notions, let for each subset $S \subseteq P$ of players (that is regarded as a coalition) and each player $j \in P$ the following sets be introduced:

$$
\begin{aligned}
I_{S \rightarrow j} & :=\left\{i \in I_{S} \mid i_{j}=\neg i_{S}\right\} \\
I_{S \rightarrow j}^{*}(B) & :=\left\{i \in I_{S \rightarrow j} \mid(B i)_{j}=i_{S}\right\}
\end{aligned}
$$

In words, $I_{S \rightarrow j}$ and $I_{S \rightarrow j}^{*}(B)$ denote the set of all inclination vectors of potential influence of coalition $S$ on player $j$, and the set of all inclination vectors of influence of $S$ on $j$ under the given influence function $B \in \mathcal{B}$, respectively.

In [23] the so-called influence indices have been defined. The general idea is to compute the weighted number of times coalition $S$ makes a player $j \in P$ change his decision. Two particular ways of weighting lead to the possibility influence index $\bar{d}(B, S \rightarrow j)$ and to the certainty influence index $\underline{d}(B, S \rightarrow j)$. The possibility index $\bar{d}(B, S \rightarrow j)$ measures the degree of influence, coalition $S$ has on player $j$, taking into account any possibility of influence. We check therefore how many inclination vectors of potential influence of coalition $S$ on player $j$ are indeed vectors of influence of $S$ on $j$. We do not verify here the inclinations of the players outside $S \cup j$. Switching to another extreme way of calculating the influence degree gives us the definition of $\underline{d}(B, S \rightarrow j)$, which measures the degree of influence, coalition $S$ has on player $j$, in another way. We take now into account only those situations in which all players outside $S \cup j$ have an inclination different from the inclination of $S$. Formal definitions of these influence indices are given below.

Definition 2.2.1 Given $B \in \mathcal{B}$, for each coalition $S \subseteq P$ of players and each player $j \in P \backslash S$, the possibility influence index of coalition $S$ on player $j$ is defined as follows:

$$
\bar{d}(B, S \rightarrow j):=\frac{\left|I_{S \rightarrow j}^{*}(B)\right|}{\left|I_{S \rightarrow j}\right|}
$$

Furthermore, by the subsequent equation the certainty influence index of coalition $S$ on player $j$ is defined:

$$
\underline{d}(B, S \rightarrow j):=\frac{\left|\left\{i \in I_{S \rightarrow j}^{*}(B) \mid \forall k \notin S: i_{k}=\neg i_{S}\right\}\right|}{2}
$$

A natural concept related to influence between players is the concept of follower. By a follower of a given coalition of players we mean a voter who always decides according to the inclination of the coalition in question. The next definition formally introduces the set of all followers.

Definition 2.2.2 Let $\emptyset \neq S \subseteq P$ be a coalition of players and $B \in \mathcal{B}$. Then the set of followers of $S$ under the influence function $B$ is defined as follows:

$$
F_{B}(S):=\left\{j \in P \mid \forall i \in I_{S}:(B i)_{j}=i_{S}\right\}
$$

Furthermore, it is worth mentioning the concept of a kernel of an influence function $B$. The kernel of $B$ is the following collection of sets:

$$
\mathcal{K}(B):=\left\{S \in 2^{P} \mid F_{B}(S) \neq \emptyset \wedge \forall S^{\prime} \in 2^{S} \backslash S: F_{B}\left(S^{\prime}\right)=\emptyset\right\}
$$

Hence, the kernel of an influence function is the set of the 'true' (minimal) influential coalitions. 


\subsection{Majority and influence by trend-setters}

In the preceding two subsections we have defined the different indices and notions dealing with coalitions, influence and followers with respect to an arbitrary influence function $B \in \mathcal{B}$ and an arbitrary group decision function $g d \in \mathcal{G}$. In practice, however, only a very small number of such functions is used.

Group decisions almost always are based on majority. This means that for each inclination vector $i \in I$ and each influence function $B \in \mathcal{B}$, the output of $g d: B(I) \rightarrow\{0,1\}$ for the decision vector $B i$ as input is 1 if the size of the set $\left\{j \in P \mid(B i)_{j}=1\right\}$ is at least $\left[\frac{n}{2}\right]+1$, where $[x]$ denotes the least natural number greater than or equal to $x$. In the remaining cases, $g d(B i)$ yields 0 as result. Instead of this so-called simple majority, in specific cases also other majority rules are used, e.g., $\frac{2}{3}$-majority or even $\frac{3}{4}$-majority.

Influences in a social network essentially are based on dependency relationships, which adequately can be modeled by a dependency graph. The vertices of such a directed loop-free graph are the players. For different players $j, k \in P$ there is an arc from $j$ to $k$ iff $j$ is a so-called trend-setter for $k$, that is, the vote of $k$ may be influenced by the inclination of $j$. Then $k$ is called a dependent player. Players without trend-setters (in terms of graph theory: the sources) are said to be independent.

Example 2.3.1. To give a concrete example, the following picture (generated with the help of RELVIEw) shows the dependency graph of a social network with a set $P$ of six players $1,2,3,4,5$ and 6 , where the vertex with label ' $k$ ' corresponds to player $k, 1 \leq k \leq 6$. Since in Sections 3 and 4 we will use this social network as running example to illustrate the developed relation-algebraic specifications, in the dependency graph also a coalition $S$ consisting of the three players $2,3,5$ is indicated by black vertices.

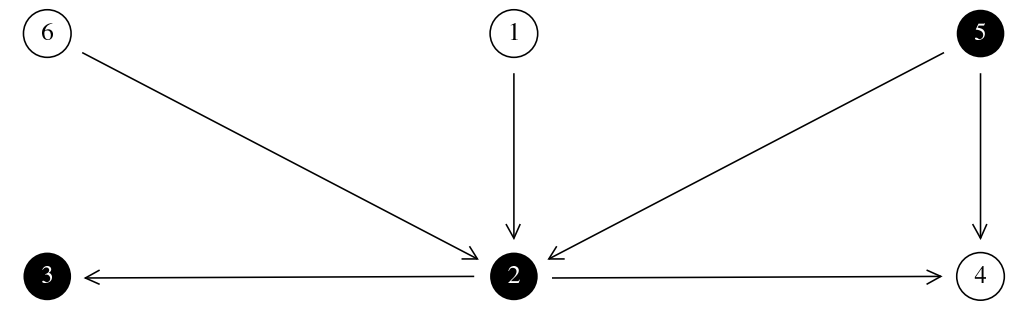

As one can see from the directed arcs of the graph, the independent players are 1,5 and 6 (no ingoing arcs), and the dependent players are 2,3 and 4 . The vote of player 2 depends on its three trendsetters (graph-theoretic predecessors) 1,5 and 6, the vote of player 3 depends on its unique trend-setter 2 , and the vote of player 4 depends on its two trend-setters 2 and 5 .

Now, assume $i \in I$ to be an inclination vector and we want to define the decision vector $B i$ in terms of the dependency graph. Of course, for an independent player $k \in P$ we are allowed to define $(B i)_{k}:=i_{k}$, i.e., to presume that he does not change his vote. On the other hand, a dependent player $k \in P$ will always follow his sole trend-setter $j \in P$ if there is exactly one. In this case, hence, we put $(B i)_{k}:=i_{j}$. It is reasonable to generalize this in such a way that a dependent player always follows his trend-setters if they have the same inclination. However, a problem appears if there are at least two trend-setters player $k \in P$ depends on, and they have different inclinations. Which trend-setter should the dependent player $k$ follow? There are several possibilities to define the influence function in such a case. Usually two possibilities are considered:

- Following only unanimous trend-setters: Here the vote of player $k$ is equal to the inclination of his trend-setters if they all have the same inclination. Otherwise, player $k$ votes according to his own inclination. 
- Following a majority of trend-setters: Here $k$ votes as the inclination of the majority of his trend-setters is. Assuming that player $k$ has $t$ trend-setters, this means that if there are at least $\left[\frac{t}{2}\right]+1$ trend-setters of $k$ with the same inclination, $k$ votes according to this inclination. Otherwise, $k$ follows his own inclination.

As in the case of group decisions, also in the second specification of the influence function via trendsetters, simple majority may be replaced by other majority rules. In the remainder of this paper, however, we restrict our analysis to simple majority in the case of the influence rule 'following a majority of trend-setters'.

\section{Relation Algebra and Modeling of Inclination Vectors}

In the first part of this section we present the facts on relation algebra that are necessary to deal with the relation-algebraic specifications and algorithms of the key concepts of Section 2. For more details on relations and relation algebra, see e.g., [47] or [11]. Next we model inclination vectors and sets of inclination vectors within relation algebra. In the last part of this section we briefly describe the RELVIEW tool.

\subsection{Relational preliminaries}

If $X$ and $Y$ are sets, then a subset $R$ of the Cartesian product $X \times Y$ is called a (binary) relation with domain $X$ and range $Y$. We denote the set (in this context also called type) of all relations with domain $X$ and range $Y$ by $[X \leftrightarrow Y]$ and write $R: X \leftrightarrow Y$ instead of $R \in[X \leftrightarrow Y]$. If $X$ and $Y$ are finite sets of size $m$ and $n$ respectively, then we may consider a relation $R: X \leftrightarrow Y$ as a Boolean matrix with $m$ rows and $n$ columns and entries from $\{0,1\}$. The Boolean matrix interpretation of relations is well suited for many purposes. For instance, it is used as one of the graphical representations of relations within the RELVIEW tool, and it is in line with the Boolean vector approach of Section 2. Therefore, in this paper we often use Boolean matrix terminology and notation. In particular, we speak about columns, rows and entries of a relation and write $R_{x, y}$ instead of $\langle x, y\rangle \in R$ or $x R y$.

We assume the reader to be familiar with the basic operations on relations, viz. $R^{\top}$ (transposition, conversion), $\bar{R}$ (complement, negation), $R \cup S$ (union, join), $R \cap S$ (intersection, meet), $R S$ (composition, multiplication), and the special relations $\mathrm{O}$ (empty relation), $\mathrm{L}$ (universal relation), and I (identity relation). If $R$ is included in $S$ we write $R \subseteq S$, and equality of $R$ and $S$ is denoted as $R=S$.

The expression $\operatorname{syq}(R, S):=\overline{R^{\top} \bar{S}} \cap \overline{\bar{R}} \bar{R}^{\top}$ is by definition the symmetric quotient syq $(R, S)$ : $Y \leftrightarrow Z$ of two relations $R: X \leftrightarrow Y$ and $S: X \leftrightarrow Z$. Many properties of this construct can be found, for example, in [47]. In the present paper, we will only use that for all $y \in Y$ and $z \in Z$ the relationship $\operatorname{syq}(R, S)_{y, z}$ holds iff for all $x \in X$ the equivalence $R_{x, y} \leftrightarrow S_{x, z}$ is valid, i.e., if the $y$-column of $R$ and the $z$-column of $S$ coincide.

Given a Cartesian product $X \times Y$ of two sets $X$ and $Y$, there are two projection functions which decompose a pair $u=\left(u_{1}, u_{2}\right)$ into its first component $u_{1}$ and its second component $u_{2}$. For a relation-algebraic approach it is useful to consider instead of these functions the corresponding projection relations $\pi: X \times Y \leftrightarrow X$ and $\rho: X \times Y \leftrightarrow Y$ such that for all pairs $u \in X \times Y$ and elements $x \in X$ and $y \in Y$ we have $\pi_{u, x}$ iff $u_{1}=x$ and $\rho_{u, y}$ iff $u_{2}=y$. Projection relations enable us to describe the well-known pairing operation of functional programming relation-algebraically as follows: For relations $R: Z \leftrightarrow X$ and $S: Z \leftrightarrow Y$ we define their pairing (frequently also called fork or tupling) $[R, S]: Z \leftrightarrow X \times Y$ by $[R, S]:=R \pi^{\top} \cap S \rho^{\top}$. Then for all $z \in Z$ and pairs $u=\left(u_{1}, u_{2}\right) \in X \times Y$ a simple reflection shows that $[R, S]_{z, u}$ iff $R_{z, u_{1}}$ and $S_{z, u_{2}}$. 


\subsection{Modeling inclination vectors and sets of inclination vectors}

Relation algebra offers some simple and elegant ways to describe subsets of a given set. For modeling influence vectors, decision vectors, and sets of followers, we will use column vectors. Following [47], these are relations $v$ (analogously to linear algebra we use lower-case letters to denote vectors) with $v=v \mathrm{~L}$. As for a column vector the range is irrelevant, we consider in the following only vectors $v: X \leftrightarrow \mathbf{1}$ with a specific singleton set $\mathbf{1}:=\{\perp\}$ as range. A column vector $v: X \leftrightarrow \mathbf{1}$ can be considered as a Boolean matrix with exactly one column, i.e., as a Boolean column vector, and it describes (or: is a description of) the subset $\left\{x \in X \mid v_{x, \perp}\right\}$ of its domain $X$. A non-empty column vector $v$ is a column point if $v v^{\top} \subseteq \mathrm{I}$, i.e., it is injective in the relational sense. This means that it represents a singleton subset of its domain or an element from it, if we identify a singleton set $\{x\}$ with the element $x$. In the Boolean matrix model, hence, a column point $v: X \leftrightarrow \mathbf{1}$ is a Boolean column vector in which exactly one entry is 1.

Vectors also allow to formalize the notions of $y$-columns and $x$-rows. E.g., for a relation $R$ : $X \leftrightarrow Y$ and $y \in Y$, the column vector $v: X \leftrightarrow \mathbf{1}$ equals the $y$-column of $R$ if for all $x \in X$ we have $v_{x, \perp}$ iff $R_{x, y}$.

For modeling kernels and subsets of the sets $I$ and $B(I)$, where the influence function $B$ is given by one of the rules 'following only unanimous trend-setters' and 'following a majority of trendsetters' of Subsection 2.3, we will use row vectors. These relations are defined as the transposes of column vectors. Again we only will need row vectors $v$ of the specific type $[\mathbf{1} \leftrightarrow Y]$ that correspond to Boolean row vectors. Then $v$ describes the subset $\left\{y \in Y \mid v_{\perp, y}\right\}$ of its range $Y$. The distinction between column vectors and row vectors is not essential. In the context of this paper, however, it is very helpful for the visualization of results of relational computations. This, hopefully, becomes clear by the subsequent continuation of our running example.

Example 3.2.1. In Example 2.3.1 we have introduced a social network with a set $P$ of six players $1,2,3,4,5$ and 6 . The following picture shows the membership relation ${ }^{1} \mathrm{M}: P \leftrightarrow 2^{P}$ between $P$ and its powerset $2^{P}$ as $6 \times 64$ Boolean RELVIEW-matrix, where a black square means a 1-entry (i.e., the relationship holds) and a white square means a 0-entry (i.e., the relationship does not hold).

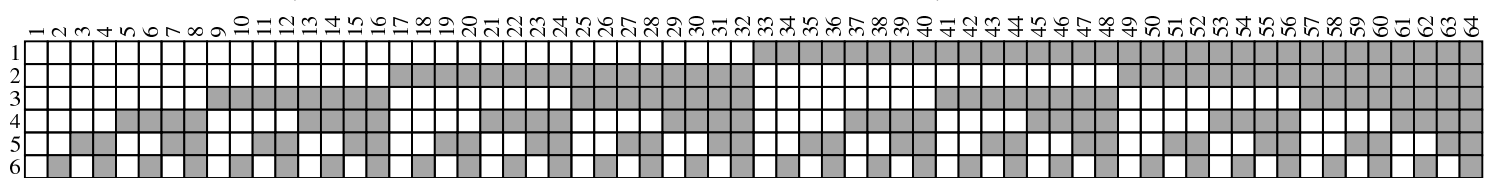

If we consider inclination vectors as relational column vectors, then this membership relation column-wisely enumerates the set $I$ of all inclination vectors, since its 64 columns exactly correspond to the 64 possible inclination vectors of the six players, and these again exactly correspond to the 64 possible subsets of the set of players. For instance, the first column corresponds to the inclination vector where each player has the inclination 'no', and the fourth column corresponds to the inclination vector where the players 5 and 6 have the inclination 'yes' and the remaining players have the inclination 'no'.

In the same way, we can obtain a $6 \times 64$ Boolean RELVIEW-matrix showing decisions of the players, where the $X$-column corresponds to the decision vector obtained from the $X$-column of $\mathrm{M}$ representing the inclination vector. Suppose, for instance, that all players are independent, that is, that we deal with the identity function, $B i=i$ for each $i \in I$.

The next RELVIEW-picture shows a row vector $m: \mathbf{1} \leftrightarrow 2^{P}$ with 64 columns that describes a subset of the powerset $2^{P}$, i.e., a subset of the set $I$ if we identify $X \in 2^{P}$ with the inclination vector $i \in I$ where exactly the players of $X$ vote 'yes'.

\footnotetext{
${ }^{1}$ A membership relation $\mathrm{M}: X \leftrightarrow 2^{X}$ relates $x \in X$ and $Y \in 2^{X}$ iff $x \in Y$. It should be emphasized that binary decision diagrams allow a very efficient implementation of $\mathrm{M}$ that uses in the worst case $3|X|+1$ BDD-vertices only. This implementation is part of RELVIEW; see [5].
} 
This row vector describes the set of the inclination vectors where the majority of the players votes 'yes'. This becomes clear if we compare the columns of both RELVIEW-pictures. Doing so, we obtain that for all $X \in 2^{P}$ the relationship $m_{\perp, X}$ holds iff the number of 1 -entries in the $X$-column of $\mathrm{M}$ is strictly larger than the number of 0 -entries in the $X$-column of $\mathrm{M}$.

Besides column vectors, row vectors and membership relations, injective (embedding) mappings are another way of modeling sets. Given a relation $\imath: Z \leftrightarrow X$, that is, an injective mapping in the relational sense of [47], $Z$ may be regarded as a subset of $X$ by identifying it with its image under $\imath$. Then the column vector $\iota^{\top} \mathrm{L}: X \leftrightarrow \mathbf{1}$ describes $Z$ in the above sense. By removing all pairs $(x, x)$ with $x \notin Z$ from the identity relation $\mathrm{I}: X \leftrightarrow X$, the transition in the other direction is also possible, that is, the construction of a relation $\operatorname{inj}(v): Z \leftrightarrow X$ from a given column vector $v: X \leftrightarrow \mathbf{1}$ describing $Z$ in such a way that $i n j(v)_{z . x}$ holds iff $z=x$ for all $z \in Z$ and $x \in X$. Such a relation is called the injective embedding generated by $v$ and is also used in our applications. Namely, if the row vector $v: \mathbf{1} \leftrightarrow 2^{P}$ describes a subset $\mathcal{S}$ of $2^{P}$ in the sense above, and $\mathrm{M}: P \leftrightarrow 2^{P}$ is the membership relation, then for all $x \in X$ and $Y \in \mathcal{S}$ we get the equivalence of $\left(\mathrm{M} i n j\left(v^{\top}\right)^{\top}\right)_{x, Y}$ and $x \in Y$. This means that the elements of $\mathcal{S}$ are described precisely by the columns of the relation $\operatorname{Minj}\left(v^{\top}\right)^{\top}: X \leftrightarrow \mathcal{S}$.

\subsection{The Kiel ReLVIEW tool}

Relation algebra has a fixed and surprisingly small set of constants and operations which (in the case of finite carrier sets) can be implemented very efficiently. Since 1993, at Kiel University we have developed a computer system for the visualization and manipulation of relations and for relational prototyping and programming, called RELVIEW. The tool is written in the C programming language and makes full use of the $\mathrm{X}$-windows graphical user interface. Details and applications can be found, for instance, in $[4,2,9]$.

RELVIEW is an interactive and graphic-oriented tool. In it all data are represented as relations which the system visualizes in two different ways. First, for relations, for which domain and range coincide, it offers a representation as directed graphs as already shown in Example 2.3.1. This includes sophisticated algorithms for drawing graphs nicely. Alternatively, as already shown in Example 3.2.1, arbitrary relations may be depicted as Boolean matrices (with, if desired, row and column labels for explanatory purposes). This second representation is very useful for visually editing and also for discovering various structural properties that are not evident from a representation of relations as directed graphs. Because RELVIEW computations frequently use very large relations, for instance, membership relations, the system uses a very efficient implementation of relations via reduced ordered binary decision diagrams. See [5] for its description.

The RELVIEw tool can manage as many relations simultaneously as memory allows and the user can manipulate and analyse them by pre-defined operations, tests and user-defined relational functions and relational programs. The pre-defined operations on relations include, for instance, ^, ,- \&, I, and * for transposition, complement, intersection, union, and composition; the relational tests include, for instance, incl, eq, and empty for testing inclusion, equality, and emptiness of relations. All that can be accessed through a lot of command buttons and simple mouse-clicks. But the usual way is to use the pre-defined operations and tests to construct relational functions and relational programs and next to evaluate the relation-algebraic expressions that are built from the relations of RELVIEW's workspace using the tool's programming language.

Relational functions are defined as it is customary in mathematics, i.e., by a function name, a list of parameters and a relation-algebraic expression. A relational program is much like a function 
procedure in the programming languages Pascal or Modula 2, except that it only uses relations as data type. It starts with a head line containing the program name and the formal parameters. Then the declaration of the local relational domains, functions, and variables follows. Domain declarations can be used to introduce projection relations and pairings of relations in the case of Cartesian products, and injection relations and sums of relations in the case of disjoint unions, respectively. The third part of a program is the body, a while-program over relations. As a program computes a value, finally, its last part consists of a return-clause, which is a relation-algebraic expression whose value after the execution of the body is the result. The following example of a RELVIEW-program Classes (formally developed in [3]) computes for an equivalence relation $R: X \leftrightarrow X$ with a set $\mathcal{C}$ of equivalence classes the canonical epimorphism from $X$ to $\mathcal{C}$ as a relation $\Phi: X \leftrightarrow \mathcal{C}$.

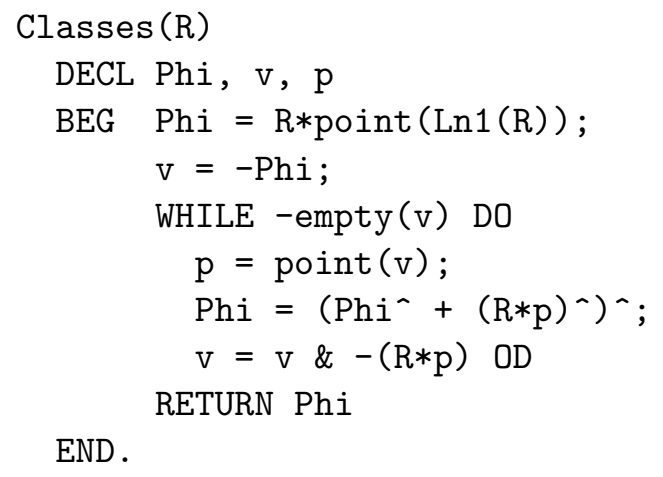

Since $\Phi$ is the relational version of the canonical epimorphism, we have for all $x \in X$ and $C \in \mathcal{C}$ that $\Phi_{x, C}$ iff $x$ belongs to $C$. Hence, if we consider the columns of the result of the RELVIEWprogram Classes as single vectors, then these vectors are pair-wise disjoint and precisely describe the elements of the set $\mathcal{C}$ in the sense explained above.

\section{Relation-algebraic Description of Measures in a Social Network}

In this section we show how the concepts introduced in Section 2 can be transformed into relationalgebraic specifications that immediately lead to RELVIEW-code. This allows to compute power indices, influence indices, sets of followers and kernels by means of the tool. We demonstrate this by depicting some of the RELVIEW-matrices and -vectors that we have obtained for our running example.

\subsection{Computing decision vectors and group decisions}

We assume a social network with a set $P$ of players. Let $D: P \leftrightarrow P$ be the relation of the dependency graph of the network. The latter property means that there is an arc from a player $j \in P$ to a player $k \in P$ iff $D_{j, k}$ holds. Then the set of the dependent players relation-algebraically is described by the column vector

$$
\operatorname{depend}(D):=D^{\top} \mathbf{L}
$$

of type $[P \leftrightarrow \mathbf{1}]$, where the used $\mathrm{L}$ has type $[P \leftrightarrow \mathbf{1}]$, too.

In Subsection 3.2 we have shown that the set $I$ of all inclination vectors immediately can be modeled by the columns of the membership relation $\mathrm{M}: P \leftrightarrow 2^{P}$. Due to this fact, in the remainder of this section we regard inclination vectors and the corresponding decision vectors as relational column vectors $i: P \leftrightarrow \mathbf{1}$ and $B i: P \leftrightarrow \mathbf{1}$, respectively. Our first goal is to develop a column-wise enumeration of the set $B(I)$ of decision vectors with relation-algebraic means, where the influence function $B$ is given by the rule 'following only unambiguous trend-setters'. As a preparatory step, we treat the transformation from $i$ to $B i$ for a single inclination vector $i$ within relation algebra. 
Theorem 4.1.1 For each inclination vector $i: P \leftrightarrow \mathbf{1}$, the decision vector $B i: P \leftrightarrow \mathbf{1}$ under the influence rule 'following only unambiguous trend-setters' is given by

$$
B i=\left(i \cap\left(\bar{d} \cup\left(d \cap D^{\top} i \cap D^{\top} \bar{i}\right)\right)\right) \cup\left(d \cap \overline{D^{\top} \bar{i}}\right),
$$

where $d:=\operatorname{depend}(D)$.

Proof Let $k \in P$ be an arbitrary player. Using the description (12) of the dependent players and $d$ as abbreviation for depend $(D)$, a formalization of the assumed rule leads to the following specification of $(B i)_{k, \perp}$ :

$$
(B i)_{k, \perp}=\left\{\begin{array}{rll}
i_{k, \perp} & : & \bar{d}_{k, \perp} \vee\left(d_{k, \perp} \wedge \exists j \in P: D_{j, k} \wedge i_{j, \perp} \wedge \exists j \in P: D_{j, k} \wedge \bar{i}_{j, \perp}\right) \\
1 & : & d_{k, \perp} \wedge \forall j \in P: D_{j, k} \rightarrow i_{j, \perp} \\
0 & : & d_{k, \perp} \wedge \forall j \in P: D_{j, k} \rightarrow \bar{i}_{j, \perp}
\end{array}\right.
$$

If we replace logical constructions by their corresponding relational counter-parts, we obtain the subsequent equivalent specification:

$$
(B i)_{k, \perp}=\left\{\begin{aligned}
i_{k, \perp} & :\left(\bar{d} \cup\left(d \cap D^{\top} i \cap D^{\top} \bar{i}\right)\right)_{k, \perp} \\
\mathrm{L}_{k, \perp} & :\left(d \cap \overline{D^{\top} \bar{i}}\right)_{k, \perp} \\
\mathrm{O}_{k, \perp} & :\left(d \cap \overline{D^{\top} i}\right)_{k, \perp}
\end{aligned}\right.
$$

Next, we transform the case distinction in the usual way with the help of disjunctions and conjunctions into a logical formula, viz.:

$$
\left(i_{k, \perp} \wedge\left(\bar{d} \cup\left(d \cap D^{\top} i \cap D^{\top} \bar{i}\right)\right)_{k, \perp}\right) \vee\left(\mathrm{L}_{k, \perp} \wedge\left(d \cap \overline{D^{\top} \bar{i}}\right)_{k, \perp}\right) \vee\left(\mathrm{O}_{k, \perp} \wedge\left(d \cap \overline{D^{\top} i}\right)_{k, \perp}\right)
$$

Since the relationship $L_{k, \perp}$ is true and the relationship $\mathrm{O}_{k, \perp}$ is false, this formula is equivalent to the following one:

$$
\left(i_{k, \perp} \wedge\left(\bar{d} \cup\left(d \cap D^{\top} i \cap D^{\top} \bar{i}\right)\right)_{k, \perp}\right) \vee\left(\left(d \cap \overline{D^{\top} \bar{i}}\right)_{k, \perp}\right)
$$

Now, we again replace in this formula logical constructions by their corresponding relational counter-parts. This yields:

$$
\left(\left(i \cap\left(\bar{d} \cup\left(d \cap D^{\top} i \cap D^{\top} \bar{i}\right)\right)\right) \cup\left(d \cap \overline{D^{\top} \bar{i}}\right)\right)_{k, \perp}
$$

If we use this formula as the right-hand side of the original specification, the definition of relational equality shows the claim.

The relation-algebraic expression $\left(i \cap\left(\bar{d} \cup\left(d \cap D^{\top} i \cap D^{\top} \bar{i}\right)\right)\right) \cup\left(d \cap \overline{D^{\top} \bar{i}}\right)$ is built from $i$ using unions, intersections, complements and left-compositions with constants (i.e., with relation-algebraic expressions free of $i$ ) only. Hence (see, for example, [6]), if we replace in it the column vector $i: P \leftrightarrow \mathbf{1}$ by the membership relation $\mathrm{M}: P \leftrightarrow 2^{P}$ that column-wisely enumerates all inclination vectors and adopt simultaneously the type $[P \leftrightarrow \mathbf{1}]$ of $d$ to the type $\left[P \leftrightarrow 2^{P}\right]$ of $\mathrm{M}$ by a right-composition with the universal row vector $\mathrm{L}: \mathbf{1} \leftrightarrow 2^{P}$, we get (with $d:=\operatorname{depend}(D)$ ) the relation

$$
\operatorname{Dvec}(D):=\left(\mathrm{M} \cap\left(\overline{d \mathbf{L}} \cup\left(d \mathrm{~L} \cap D^{\top} \mathrm{M} \cap D^{\top} \overline{\mathrm{M}}\right)\right)\right) \cup\left(d \mathrm{~L} \cap \overline{D^{\top} \overline{\mathrm{M}}}\right)
$$

of type $\left[P \leftrightarrow 2^{P}\right]$ that column-wisely enumerates the set $B(I)$ of decision vectors. The latter property means: For all $X \in 2^{P}$, if the $X$-column of M equals $i: P \leftrightarrow \mathbf{1}$ then, under the assumed rule, the $X$-column of $\operatorname{Dvec}(D)$ equals $B i: P \leftrightarrow \mathbf{1}$. 
Having obtained a relation-algebraic specification for the column-wise enumeration of the decision vectors, our next goal is to obtain with the help of (13) a relation-algebraic specification of the group decisions under majority as decision rule via a row vector. To reach the goal, we assume that a row vector $m: \mathbf{1} \leftrightarrow 2^{P}$ is available such that for all $X \in 2^{P}$ we have $m_{\perp, X}$ iff $|X| \geq\left[\frac{|P|}{2}\right]+1$. In RELVIEW such a vector can be easily obtained with the help of the base operation cardfilter ${ }^{2}$ as

$$
m:=\overline{\operatorname{cardfilter}(\mathrm{L}, w)}^{\mathrm{T}} \text {, }
$$

where the first argument $\mathrm{L}: 2^{P} \leftrightarrow \mathbf{1}$ describes the entire powerset $2^{P}$, and the second argument $w: W \leftrightarrow \mathbf{1}$ determines the threshold for majority by its length, i.e., fulfils $|W|=\left[\frac{|P|}{2}\right]+1$. Based on (14), we can specify the desired row vector as shown now.

Theorem 4.1.2 Let, based on the specifications (13) and (14), the row vector gdv(D) of type $\left[\mathbf{1} \leftrightarrow 2^{P}\right]$ be defined by

$$
g d v(D):=m \operatorname{syq}(\mathrm{M}, \operatorname{Dvec}(D))
$$

where $\mathrm{M}: P \leftrightarrow 2^{P}$ is the membership relation. Then we have for all $X \in 2^{P}$ : If the decision vector $B i: P \leftrightarrow \mathbf{1}$ equals the $X$-column of Dvec $(D)$, then $g d v(D)_{\perp, X}$ holds iff the number of 1-entries in $B i$ is at least $\left[\frac{|P|}{2}\right]+1$.

Proof We calculate as given below, where the assumption that the $X$-column of $\operatorname{Dvec}(D)$ equals $B i$ is used in the last step, and the inclination vector $i^{(Y)}$ introduced in this step coincides with the $Y$-column of $\mathrm{M}$.

$$
\begin{aligned}
g d v(D)_{\perp, X} & \Longleftrightarrow(m \operatorname{syq}(\mathrm{M}, \operatorname{Dvec}(D)))_{\perp, X} \\
& \Longleftrightarrow \exists Y \in 2^{P}: m_{\perp, Y} \wedge \operatorname{syq}(\mathrm{M}, \operatorname{Dvec}(D))_{Y, X} \\
& \Longleftrightarrow \exists Y \in 2^{P}: m_{\perp, Y} \wedge\left(\forall k \in P: \mathrm{M}_{k, Y} \leftrightarrow \operatorname{Dvec}(D)_{k, X}\right) \\
& \Longleftrightarrow \exists Y \in 2^{P}:|Y| \geq\left[\frac{|P|}{2}\right]+1 \wedge\left(\forall k \in P: \mathrm{M}_{k, Y} \leftrightarrow \operatorname{Dvec}(D)_{k, X}\right) \\
& \Longleftrightarrow \exists Y \in 2^{P}:|Y| \geq\left[\frac{|P|}{2}\right]+1 \wedge i^{(Y)}=B i
\end{aligned}
$$

Now the claim follows from the simple fact that the number of 1-entries in the column vector $i^{(Y)}$ equals $|Y|$.

Summing up, we have for the influence function $B$ defined by the rule 'following only unambiguous trend-setters' and for the group decision function $g d$ defined by simple majority: If the inclination vector $i: P \leftrightarrow \mathbf{1}$ is given by the $X$-column of the membership relation $\mathrm{M}: P \leftrightarrow 2^{P}$, then the corresponding decision vector $B i: P \leftrightarrow \mathbf{1}$ is given by the $X$-column of the relation $\operatorname{Dvec}(D): P \leftrightarrow 2^{P}$ and, furthermore, $g d(B i)=1$ iff $g d v(D)_{\perp, X}$ holds.

Example 4.1.1. We have transformed the above relation-algebraic specifications into RELVIEWcode. To give examples how such programs look like, we present in the following the code for both specifications. In the following RELVIEW-programs Dvec and $g d v$ the calls epsi $(O(D))$ of the predefined operation epsi compute the membership relation $\mathrm{M}: P \leftrightarrow 2^{P}$, and the calls $\operatorname{Lin}(M)$ of the

\footnotetext{
${ }^{2}$ If $v: 2^{M} \leftrightarrow 1$ represents the subset $\mathcal{S}$ of $2^{M}$ and the size of the domain of $w: W \leftrightarrow \mathbf{1}$ is at most $|M|+1$, then for all $X \in 2^{M}$ we have $\operatorname{cardfilter}(v, w)_{X, \perp}$ iff $X \in \mathcal{S}$ and $|X|<|W|$. Hence, the complement of cardfilter $(\mathrm{L}, w)$ represents the subset of $2^{M}$ whose elements have at least size $|W|$.
} 
pre-defined operation $L 1 n$ yield the row vector $L: 1 \leftrightarrow 2^{P} .3$

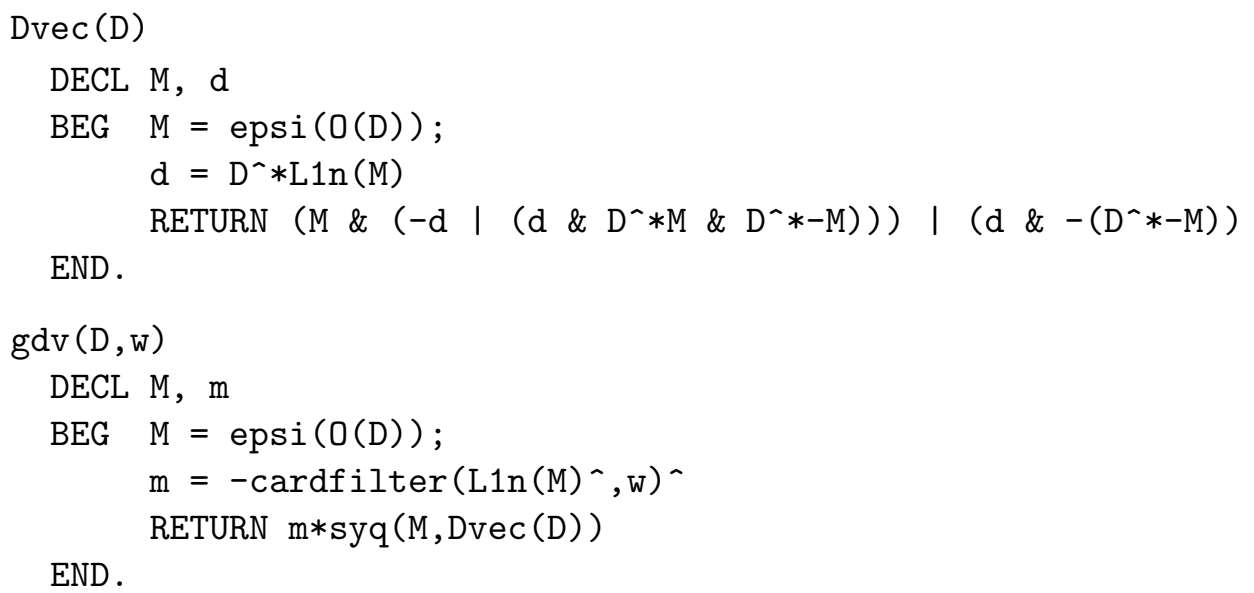

Applied to the relation $D$ of our running example and a column vector $w$ of length 4 (the threshold of majority) in the case of the second program, we obtained by their means the following results for $\operatorname{Dvec}(D)$ and $g d v(D)$. The 64 columns of the $6 \times 64$ RELVIEW-matrix represent the 64 decision vectors obtained from the 64 inclination vectors, and the entries of the $1 \times 64$ row vector below this matrix indicate the group decision for each decision vector.

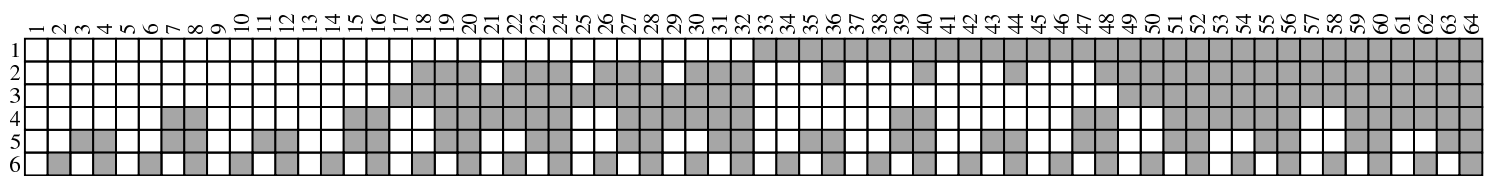

Let us explain these results by the specific inclination vectors treated in Example 3.2.1. For the first column of the membership relation M of Example 3.2.1, where each player votes 'no', we obtain 'no' also as decision of each player as well as of the entire group. The same is the case if the inclination of the players 5 and 6 is 'yes' and that of the remaining players is 'no'; cf. the fourth columns of $\mathrm{M}, \operatorname{Dvec}(D)$ and $g d v(D)$.

We also have developed a RELVIEW-program that computes the column-wise enumeration of the decision vectors under 'following a majority of the trend-setters' as the influence rule by handling one after another the columns of the membership relation via a loop. If we use this program in the case of our running example, we obtain the following RELVIEW-matrix and row vector for the decision vectors and the group decisions, respectively.

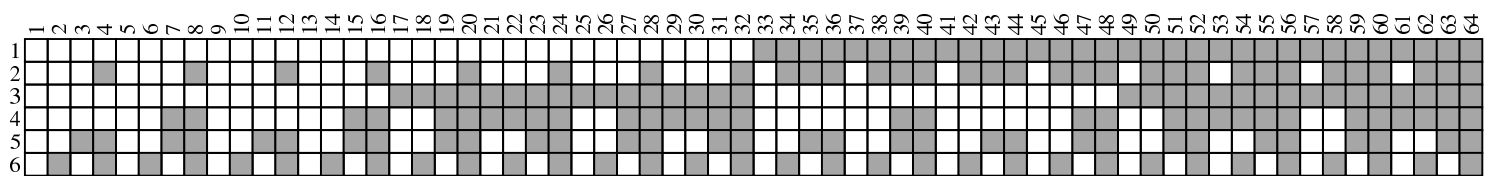

In contrast with the influence rule 'following only unambiguous trend-setters', now the inclinations 'yes' of the players 5 and 6 and 'no' of the remaining players yield a decision, where player 2 changes

\footnotetext{
${ }^{3}$ We need $n$ and 1 in the pre-defined RELVIEW-operations for typing. If $R: X \leftrightarrow Y$ is a relation, then $L(R)$ yields the universal relation $L$ of type $[X \leftrightarrow Y], L n 1(R)$ yields the universal column vector $L$ of type $[X \leftrightarrow \mathbf{1}]$, and $\operatorname{L1n}(R)$ yields the universal row vector $\mathrm{L}$ of type $[\mathbf{1} \leftrightarrow Y]$. Hence, in the RELVIEW-programs $\operatorname{Lin}(M)$ is the universal row vector of type $\left[\mathbf{1} \leftrightarrow 2^{P}\right]$ and so its transposition the universal column vector of type $\left[2^{P} \leftrightarrow \mathbf{1}\right]$. The RELVIEW-operation cardfilter works on column-vectors; that is the only reason for the transposition here.
} 
his opinion from 'no' to 'yes', because of the 'yes'-vote of the majority of the trend-setters player 2 depends on. In spite of this change, the group's decision remains 'no'.

An example where the different influence rules yield different group decisions for the same inclination vector is given by the 8th columns of the matrices and row vectors, respectively. If the inclination of the players 4,5 and 6 is 'yes' and that of the remaining players is 'no', then 'following only unambiguous trend-setters' implies 'inclination equals decision' and the group decision 'no'. Nevertheless, 'following a majority of the trend-setters' implies that also player 2 finally votes 'yes', so that the collective vote becomes 'yes', too.

As we can see from the matrices of this example, within the column-wise enumeration of the decision vectors $E:=\operatorname{Dvec}(D)$ some columns may occur several times. But it is very easy to obtain a representation without multiple columns. We only have to compute the canonical epimorphism $\Phi$ of the equivalence relation syq $(E, E)$ via the relational program Classes of Subsection 3.3. Then $E \Phi$ yields the desired result.

\subsection{Computing power indices}

Now, we demonstrate how to compute the indices presented in Subsection 2.1 with relation-algebraic means. The main steps are to determine four row vectors of type $\left[\mathbf{1} \leftrightarrow 2^{P}\right]$ which describe the four sets $I_{k}^{++}, I_{k}^{+-}, I_{k}^{-+}$and $I_{k}^{--}$, respectively. Since RELVIEW yields for each computed relation also the number of its 1-entries (i.e., its set-theoretic size), from the vector descriptions we get the numbers $\left|I_{k}^{++}\right|,\left|I_{k}^{+-}\right|,\left|I_{k}^{-+}\right|$and $\left|I_{k}^{--}\right|$, and from these also the various power indices using straightforwardly their specifications of Subsection 2.1. Note, that the set $I^{+}$used in the definition of the indices $\mathrm{M}_{1} \mathrm{GHB}_{k}, \mathrm{M}_{4} \mathrm{GHB}_{k}$ and MGHB is already described by the row vector $g d v(D)$ of Theorem 4.1.2 or its analogon in the case of the rule 'following a majority of the trend-setters'.

We assume that the player $k \in P$, on which the four sets $I_{k}^{++}, I_{k}^{+-}, I_{k}^{-+}$and $I_{k}^{--}$depend, is described by a column point $p: P \leftrightarrow \mathbf{1}$ in the relational sense. As the definitions of the sets also use the values $g d(B i)$ for $i \in I$, we assume, furthermore, that the group decision row vector $g:=g d v(D)$ is at hand (where the influence rule used for its computation is arbitrary). Then we are able to prove the following result.

Theorem 4.2.1 Let, depending on the column point $p: P \leftrightarrow \mathbf{1}$ and the row vector $g: \mathbf{1} \leftrightarrow 2^{P}$, the four vectors ipp $(p, g)$, ipm $(p, g)$, imp $(p, g)$ and $i m m(p, g)$ of type $\left[\mathbf{1} \leftrightarrow 2^{P}\right]$ be defined as follows, where $\mathrm{M}: P \leftrightarrow 2^{P}$ is the membership relation:

$$
\begin{array}{rlrl}
i p p(p, g) & :=p^{\top} \mathrm{M} \cap g & i p m(p, g) & :=p^{\top} \mathrm{M} \cap \bar{g} \\
i m p(p, g) & :=p^{\top} \overline{\mathrm{M}} \cap g & i m m(p, g) & :=p^{\top} \overline{\mathrm{M}} \cap \bar{g}
\end{array}
$$

Then we have for all $X \in 2^{P}$ : If the $X$-column of $\mathrm{M}$ equals the inclination vector $i: P \leftrightarrow \mathbf{1}$, then we have that $\operatorname{ipp}(p, g)_{\perp, X}$ holds iff $i \in I_{k}^{++}, \operatorname{ipm}(p, g)_{\perp, X}$ holds iff $i \in I_{k}^{+-}$, imp $(p, g)_{\perp, X}$ holds iff $i \in I_{k}^{-+}$, and $i m m(p, g)_{\perp, X}$ holds iff $i \in I_{k}^{--}$.

Proof The first claim follows from

$$
\begin{aligned}
i p p(p, g)_{\perp, X} & \Longleftrightarrow\left(p^{\top} \mathrm{M} \cap g\right)_{\perp, X} & & \\
& \Longleftrightarrow \exists j \in P: p_{j, \perp} \wedge \mathrm{M}_{j, X} \wedge g_{\perp, X} & & \\
& \Longleftrightarrow \exists j \in P: j=k \wedge \mathrm{M}_{j, X} \wedge g_{\perp, X} & & p \text { describes } k \\
& \Longleftrightarrow \mathrm{M}_{k, X} \wedge g_{\perp, X} & & g d(B i)=1 \text { iff } g d v(D)_{\perp, X} \\
& \Longleftrightarrow i_{k, \perp} \wedge g d(B i)=1 & & \text { assumption } \\
& \Longleftrightarrow i \in I_{k}^{++} & &
\end{aligned}
$$


since the relationship $i_{k, \perp}$ is nothing else than $i_{k}=1$ for the $k$-component of a Boolean vector in the sense of Section 2. In the same way the remaining equivalences can be calculated.

Due to this theorem, the row vector $i p p(p, g)$ precisely designates those columns of the membership relation $\mathrm{M}$ which belong to the set $I_{k}^{++}$, and the remaining three row vectors of the theorem do the same for the three sets $I_{k}^{+-}, I_{k}^{-+}$and $I_{k}^{--}$, respectively. Once more it is very easy to translate the relation-algebraic specifications of Theorem 4.2.1 into the programming language of RELVIEW. Subsequently, we show some results for our running example. We restrict our analysis to the HoedeBakker index defined in (1).

Example 4.2.1. In the following, we concentrate on player 2 which is influenced by the three trend-setters 1, 5 and 6 . Using 'following only unambiguous trend-setters' as influence rule, the first row of the following $2 \times 64$ RELVIEW-matrix depicts the row vector $i p p(p, g)$, i.e., precisely designates those columns of the membership relation $M: P \leftrightarrow 2^{P}$ that belong to the set $I_{2}^{++}$. The second row of the matrix does the same for $I_{2}^{+-}$.

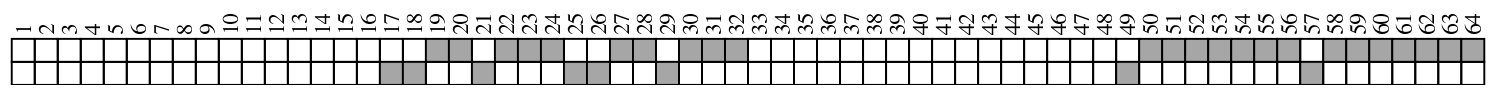

Counting the 1-entries of the single rows, we obtain that in 24 cases the inclination 'yes' of player 2 coincides with the group decision 'yes', and in 8 cases the inclination 'yes' of player 2 is opposed to the group decision 'no'. Hence, in the social network of the example, the Hoede-Bakker index for player 2 is $\frac{1}{32}(24-8)=0.5$.

The next picture shows, again by means of one matrix, the RELVIEW-representations of the two sets $I_{2}^{++}$(first row) and $I_{2}^{+-}$(second row) of the inclination vectors with 'following the majority of the trend-setters' as the influence rule.

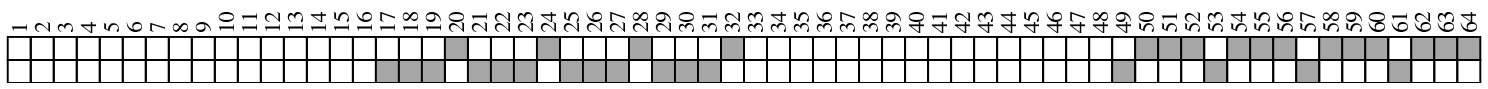

Counting the 1-entries of the single rows, we obtain here that in 16 cases player 2's inclination and the group vote are 'yes', and in the same number of cases player 2 has the inclination 'yes', but the group says 'no'. Under this rule, hence, we have $\frac{1}{32}(16-16)=0$ as the Hoede-Bakker index for player 2 .

We have also computed the Hoede-Bakker indices for the other players. In the case of the influence rule 'following only unambiguous trend-setters', we have obtained that not an independent player, but player 2 is the most powerful player of the network in our running example. For the independent players we have obtained (in the case of both rules) the Hoede-Bakker indices 0.125 (players 1 and 6) and 0.25 (player 5). This result agrees with observations that frequently can be made in practice, e.g., if the network is given by a hierarchic administration structure. If players may obtain instructions from more than one 'superior' player, then not the independent players (the big bosses) are the most powerful ones, but those in the middle of the hierarchy.

\subsection{Computing influence indices, followers and kernels}

In the following, we assume a coalition $S$ of players to be described by a column vector $s: P \leftrightarrow \mathbf{1}$, and a single player $j \in P$ to be described by a column point $p: P \leftrightarrow \mathbf{1}$. We want to compute the possibility influence index of $S$ on player $j$. Since it is defined by means of the sizes of the sets $I_{S \rightarrow j}$ and $I_{S \rightarrow j}^{*}(B)$, our task is to describe these sets within relation algebra. A translation of the results into RELVIEW-code then allows to proceed exactly as in the case of the power indices.

Both, $I_{S \rightarrow j}$ and $I_{S \rightarrow j}^{*}(B)$ are subsets of $I_{S}$. Therefore, as a preparatory step we describe the latter set of inclination vectors with relation-algebraic means. Doing so, projection relations and the pairing operation come into the play. 
Theorem 4.3.1 Assume $s: P \leftrightarrow 1$ as description of the coalition $S \subseteq P$ and the row vector is $(s)$ of type $\left[\mathbf{1} \leftrightarrow 2^{P}\right]$ to be defined as

$$
i s(s):=\left[s^{\top}, s^{\top}\right] \overline{(\overline{\pi \mathrm{M}} \cup \rho \mathrm{M}) \cap(\overline{\rho \mathrm{M}} \cup \pi \mathrm{M})},
$$

where $\mathrm{M}: P \leftrightarrow 2^{P}$ is the membership relation, and $\pi: P \times P \leftrightarrow P$ and $\rho: P \times P \leftrightarrow P$ are the projection relations in the sense of Subsection 3.1. Then we have for all $X \in 2^{P}:$ If the $X$-column of $\mathrm{M}$ equals the inclination vector $i: P \leftrightarrow \mathbf{1}$, then is $(s)_{\perp, X}$ holds iff $i \in I_{S}$.

Proof Since the $X$-column of $\mathrm{M}$ equals $i$, we have for all pairs $u=\left(u_{1}, u_{2}\right) \in P \times P$ the following equivalence:

$$
\begin{aligned}
i_{u_{1}, \perp}=i_{u_{2}, \perp} & \Longleftrightarrow \mathrm{M}_{u_{1}, X} \leftrightarrow \mathrm{M}_{u_{2}, X} \\
& \Longleftrightarrow(\pi \mathrm{M})_{u, X} \leftrightarrow(\rho \mathrm{M})_{u, X} \\
& \Longleftrightarrow\left((\pi \mathrm{M})_{u, X} \rightarrow(\rho \mathrm{M})_{u, X}\right) \wedge\left((\rho \mathrm{M})_{u, X} \rightarrow(\pi \mathrm{M})_{u, X}\right) \\
& \Longleftrightarrow\left(\overline{\pi \mathrm{M}}_{u, X} \vee(\rho \mathrm{M})_{u, X} \wedge\left(\overline{\rho \mathrm{M}}_{u, X} \vee(\pi \mathrm{M})_{u, X}\right)\right. \\
& \Longleftrightarrow((\overline{\pi \mathrm{M}} \cup \rho \mathrm{M}) \cap(\overline{\rho \mathrm{M}} \cup \pi \mathrm{M}))_{u, X}
\end{aligned}
$$

From this result and since $s$ describes $S$, we obtain

$$
\begin{aligned}
& i s(s)_{\perp, X} \Longleftrightarrow\left(\left[s^{\top}, s^{\top}\right] \overline{(\overline{\pi \mathrm{M}} \cup \rho \mathrm{M}) \cap(\overline{\rho \mathrm{M}} \cup \pi \mathrm{M})}\right)_{\perp, X}
\end{aligned}
$$

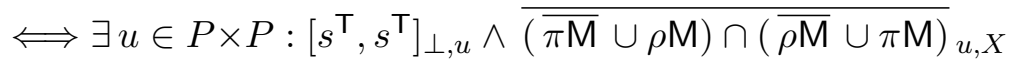

$$
\begin{aligned}
& \Longleftrightarrow \forall u \in P \times P:\left[s^{\top}, s^{\top}\right]_{\perp, u} \rightarrow((\overline{\pi \mathrm{M}} \cup \rho \mathrm{M}) \cap(\overline{\rho \mathrm{M}} \cup \pi \mathrm{M}))_{u, X} \\
& \Longleftrightarrow \forall u \in P \times P: s_{u_{1}, \perp} \wedge s_{u_{2}, \perp} \rightarrow\left(i_{u_{1}, \perp}=i_{u_{2}, \perp}\right) \\
& \Longleftrightarrow \forall u \in P \times P: u_{1} \in S \wedge u_{2} \in S \rightarrow\left(i_{u_{1}, \perp}=i_{u_{2}, \perp}\right)
\end{aligned}
$$

The latter formula of this calculation exactly says that $i \in I_{S}$.

Hence, the row vector $i s(s)$ precisely designates those columns of the membership relation $\mathrm{M}$ which belong to the set $I_{S}$. Next, we attack the relation-algebraic specification of the set $I_{S \rightarrow j}$, where $j \in P$ is described by the column point $p: P \leftrightarrow \mathbf{1}$. In the following theorem we relation-algebraically specify a row vector that precisely designates those columns of $\mathrm{M}$ which are inclination vectors of potential influence of $S$ on $j$.

Theorem 4.3.2 Assume $s: P \leftrightarrow \mathbf{1}$ to describe the coalition $S \subseteq P$, the column point $p: P \leftrightarrow \mathbf{1}$ to describe the player $j \in P$, the column point $q \subseteq s$ to describe some player $k \in S$, and the row vector potinf $(s, p)$ of type $\left[\mathbf{1} \leftrightarrow 2^{P}\right]$ to be defined as

$$
\operatorname{potinf}(s, p):=\left(\left(r \cup r^{\prime}\right) \cap \overline{r \cap r^{\prime}}\right) \operatorname{inj}\left(i s(s)^{\top}\right),
$$

where $r:=p^{\top} \mathrm{M} i n j\left(i s(s)^{\top}\right)^{\top}$ and $r^{\prime}:=q^{\top} \mathrm{M} i n j\left(i s(s)^{\top}\right)^{\top}$ with $\mathrm{M}: P \leftrightarrow 2^{P}$ as membership relation. Then we have for all $X \in 2^{P}$ : If the $X$-column of $\mathrm{M}$ equals the inclination vector $i: P \leftrightarrow \mathbf{1}$, then potinf $(s, p)_{\perp, X}$ holds iff $i \in I_{S \rightarrow j}$.

Proof From Theorem 4.3.1 we know that the row vector is $(s)$ describes the subset $\mathcal{S}$ of $2^{P}$ that consists of those sets $Y \in 2^{P}$ for which the $Y$-column of $\mathrm{M}$ is, considered as inclination vector, a member of $I_{S}$. Furthermore, $\operatorname{inj}\left(i s(s)^{\top}\right): \mathcal{S} \leftrightarrow 2^{P}$ is the relational description of the identity 
mapping from $\mathcal{S}$ to $2^{P}$; see Subsection 3.2. Using these facts and the assumption that the $X$ column of $\mathrm{M}$ equals $i$, we get

$$
\begin{aligned}
\operatorname{potinf}(s, p)_{\perp, X} & \Longleftrightarrow\left(\left(\left(r \cup r^{\prime}\right) \cap \overline{r \cap r^{\prime}}\right) \operatorname{inj}\left(i s(s)^{\top}\right)\right)_{\perp, X} \\
& \Longleftrightarrow \exists Y \in \mathcal{S}:\left(\left(r \cup r^{\prime}\right) \cap \overline{r \cap r^{\prime}}\right)_{\perp, Y} \wedge \operatorname{inj}\left(i s(s)^{\top}\right)_{Y, X} \\
& \Longleftrightarrow \exists Y \in \mathcal{S}:\left(\left(r \cup r^{\prime}\right) \cap \overline{r \cap r^{\prime}}\right)_{\perp, Y} \wedge Y=X \\
& \Longleftrightarrow X \in \mathcal{S} \wedge\left(\left(r \cup r^{\prime}\right) \cap \overline{r \cap r^{\prime}}\right)_{\perp, X} \\
& \Longleftrightarrow i \in I_{S} \wedge\left(\left(r \cup r^{\prime}\right) \cap \overline{r \cap r^{\prime}}\right)_{\perp, X}
\end{aligned}
$$

Next, we apply that the column point $p$ describes the player $j \in P$, again we apply the assumption and get in the case $X \in \mathcal{S}$ the equivalence

$$
r_{\perp, X} \Longleftrightarrow \exists l \in P: p_{l, \perp} \wedge \mathrm{M}_{l, X} \Longleftrightarrow \exists l \in P: j=l \wedge l \in X \Longleftrightarrow j \in X \Longleftrightarrow i_{j, \perp} .
$$

In the same way ${ }^{4}$ from the description of $k \in P$ by the column vector $q$ and the assumption we obtain that $r_{\perp, X}^{\prime}$ is equivalent to $i_{k, \perp}$, i.e., to the $k$-entry of $i$ to be 1 . The latter fact implies the equivalence of $r_{\perp, X}^{\prime}$ and $i_{S}=1$ for the Boolean value $i_{S}$ used in the specification of $I_{S \rightarrow j}$. A consequence of the just shown properties is

$$
\begin{aligned}
\left(\left(r \cup r^{\prime}\right) \cap \overline{r \cap r^{\prime}}\right)_{\perp, X} & \Longleftrightarrow\left(r_{\perp, X} \vee r_{\perp, X}^{\prime}\right) \wedge \neg\left(r_{\perp, X} \wedge r_{\perp, X}^{\prime}\right) \\
& \Longleftrightarrow\left(i_{j, \perp} \vee i_{S}=1\right) \wedge \neg\left(i_{j, \perp} \wedge i_{S}=1\right) \\
& \Longleftrightarrow\left(i_{j, \perp} \leftrightarrow \neg\left(i_{S}=1\right)\right) \\
& \Longleftrightarrow\left(i_{j}=\neg i_{S}\right)
\end{aligned}
$$

since again the relationship $i_{j, \perp}$ is nothing else than the validity of $i_{j}=1$ in the sense of Section 2 . Now, a combination of this fact with the result of the above calculation yields

$$
\operatorname{potinf}(s, p)_{\perp, X} \Longleftrightarrow i \in I_{S} \wedge\left(i_{j}=\neg i_{S}\right)
$$

and this, finally, shows the claim.

To obtain a row vector $\inf (s, p, D)$ of type $\left[\mathbf{1} \leftrightarrow 2^{P}\right]$ that precisely designates those columns of the membership relation $\mathrm{M}: P \leftrightarrow 2^{P}$ which are inclination vectors of influence of $S$ on $j$, i.e., members of $I_{S \rightarrow j}^{*}(B)$, we use the equation

$$
I_{S \rightarrow j}^{*}(B)=I_{S \rightarrow j} \cap\left\{i \in I_{S} \mid(B i)_{j}=i_{S}\right\} .
$$

The definition of the set $\left\{i \in I_{S} \mid(B i)_{j}=i_{S}\right\}$ is rather similar to that of the set $I_{S \rightarrow j}$; cf. Subsection 2.2. Compared with the latter one, only the expressions $B i$ and $i_{S}$ are used instead of $i$ and $\neg i_{S}$. This immediately leads to the relation-algebraic specification of the set $I_{S \rightarrow j}^{*}(B)$ by the row vector

$$
\inf (s, p, D):=\operatorname{potinf}(s, p) \cap \overline{\left(r \cup r^{\prime}\right) \cap \overline{r \cap r^{\prime}}} \operatorname{inj}\left(i s(s)^{\top}\right)
$$

with now $r$ and $r^{\prime}$ given by $r:=p^{\top} \operatorname{Dvec}(D) \operatorname{inj}\left(i s(s)^{\top}\right)^{\top}$ and $r^{\prime}:=q^{\top} \mathrm{M} i n j\left(i s(s)^{\top}\right)^{\top}$ (see Theorem 4.3.2). This is due to the fact that the decision vectors column-wisely are enumerated via the relation $\operatorname{Dvec}(D): P \leftrightarrow 2^{P}$ (where the concrete influence rule is irrelevant) and, for the inclination vector $i: P \leftrightarrow \mathbf{1}$ being the $X$-column of the membership relation $\mathrm{M}: P \leftrightarrow 2^{P}$, the relationship $\left(\left(r \cup r^{\prime}\right) \cap \overline{r \cap r^{\prime}}\right)_{\perp, X}$ does not hold iff $i_{j, \perp}$ and $i_{S}=1$ are equivalent.

In the following, we demonstrate by means of our running example how results of the RELVIEW-programs look like that immediately are obtained from the just developed relation-algebraic specifications by writing them in the programming language of the tool.

\footnotetext{
${ }^{4}$ In terms of matrices, $r$ equals the $j$-row of $\mathrm{M} i n j\left(i s(s)^{\top}\right)^{\top}$ and $r^{\prime}$ the $k$-row of the same relation.
} 
Example 4.3.1. Let us consider the coalition $S$ with players 2,3 and 5, that is in the dependency graph of Example 2.3.1 indicated by black vertices. For this coalition, the set $I_{S}$ contains 16 inclination vectors. This follows from the following two RELVIEW-pictures. The first one shows again the membership relation $\mathrm{M}: P \leftrightarrow 2^{P}$ of Example 3.2.1 and the second one the row vector $i s(s): \mathbf{1} \leftrightarrow 2^{P}$, where the column vector $s: P \leftrightarrow \mathbf{1}$ describes $S$.

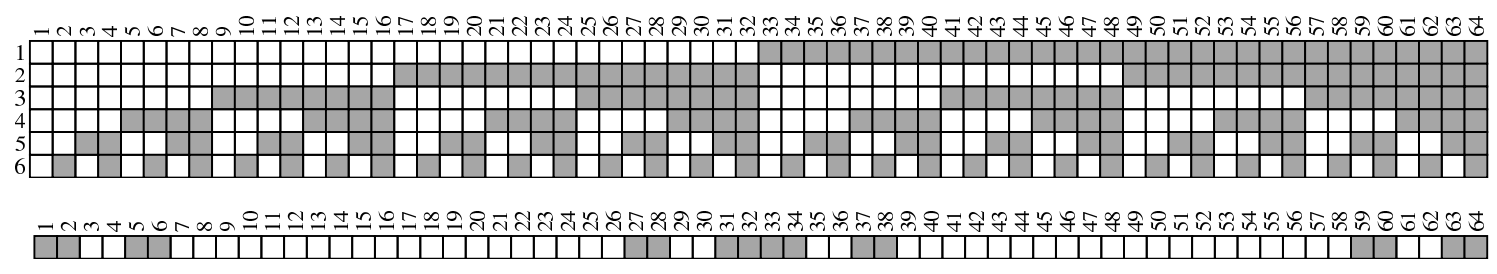

The row vector precisely designates those columns of the matrix where the entries 2,3 and 5 have the same colour.

Below we show the RELVIEW-representations of the sets $I_{S \rightarrow j}$ and $I_{S \rightarrow j}^{*}(B)$ for those players $j$ which are not contained in the coalition $S$. The first row of the following $2 \times 64$ RELVIEW-matrix indicates the columns of the membership relation $\mathrm{M}$ which are inclination vectors from set $I_{S \rightarrow 1}$, and the second row indicates the inclination vectors that belong to $I_{S \rightarrow 1}^{*}(B)$, where 'following only unambiguous trend-setters' is the influence rule. The next two RELVIEW-matrices do the same for the sets $I_{S \rightarrow 4}$ and $I_{S \rightarrow 4}^{*}(B)$ and the sets $I_{S \rightarrow 6}$ and $I_{S \rightarrow 6}^{*}(B)$, respectively. From the three pictures we obtain that, under the assumed rule, the possibility influence indices of $S$ on the players 1 and 6 are 0 , and the possibility influence index of $S$ on the player 4 is 1 .

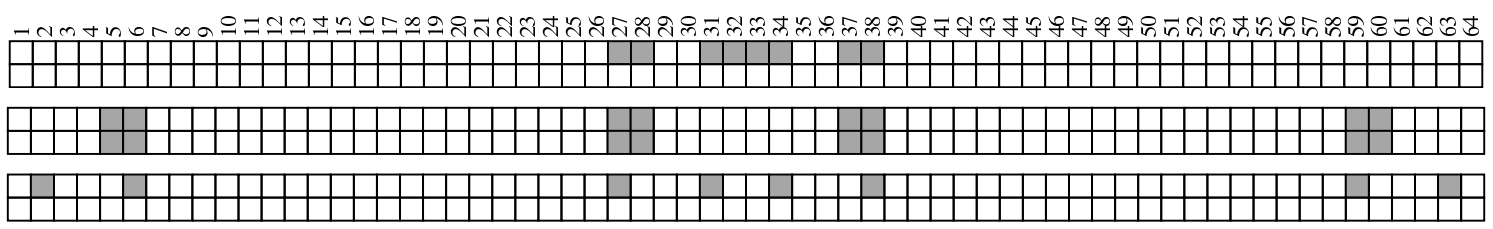

The next three RELVIEW-matrices are analogous to the just presented ones, however, with 'following the majority of the trend-setters' as influence rule for the computation of the decision vectors.

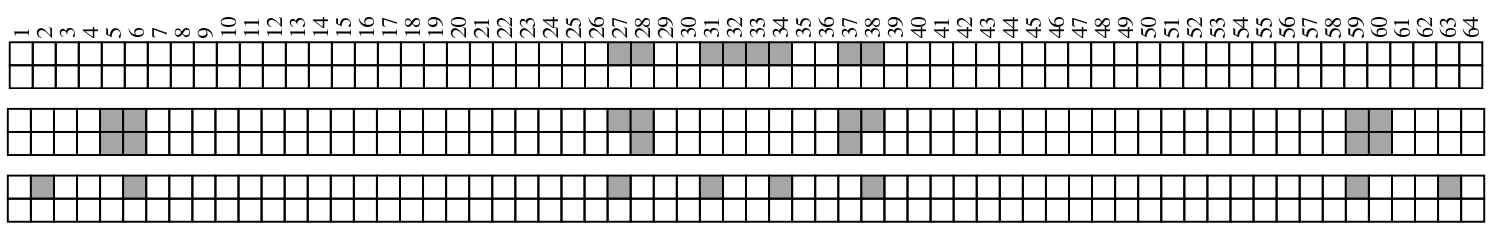

Comparing these matrices with the above ones, we get that in the case of our running example both influence rules lead to the same sets and, hence, the same indices. ${ }^{5}$ In words, the results say: Whatever of the two influence rules is applied, the coalition $S$ is without any influence on the players 1 and 6 and in the case of player 4 there is a possibility of influence of $S$ on 4 and it is even maximal.

From $I_{S \rightarrow 1}^{*}(B)=I_{S \rightarrow 6}^{*}(B)=\emptyset$ in the case of both influence rules we immediately get that the certainty influence index of $S$ on player 1 as well as on player 6 is 0 . The next ReLVIEWpicture shows the column-wise enumeration of the set $I_{S \rightarrow 4}^{*}(B)$ (which is equal under both rules) as computed by the tool. To enhance readability, the used column labels correspond to the column labels of the above membership relation.

\footnotetext{
${ }^{5}$ It should be mentioned that the equal results for player $k:=4$ in our running example are caused by the fact that it has exactly two trend-setters. In such a case the value $(B i)_{k}$ computed from $i$ via 'following only unambiguous trend-setters' is the same as that computed via 'following the majority of the trend-setters'. To give an example where both rules lead to different results, we want to mention that, for $S^{\prime}:=\{3,5\}$ and player 2 , we get $\left|I_{S^{\prime} \rightarrow 2}\right|=16$ and $\left|I_{S^{\prime} \rightarrow 2}^{*}(B)\right|=4$ if $B$ is given by 'following only unambiguous trend-setters', respectively $\left|I_{S^{\prime} \rightarrow 2}^{*}(B)\right|=12$ if $B$ is given by 'following the majority of the trend-setters'.
} 


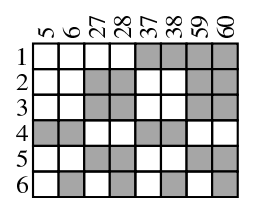

Under both rules for $S$ and player 4 exactly 2 of the eight inclination vectors $i \in I_{S \rightarrow 4}^{*}(B)$ fulfil the property $i_{k}=\neg i_{S}$ for all players $k$ not from $S$. The first one is labeled with 27 (here $i_{S}=1$ and $i_{k}=0$ for $k=1,4,6$ ), and the second one is labeled with 38 (and is the complement of the first one). Hence, the certainty influence index $\underline{d}(B,\{2,3,5\} \rightarrow 4)$ is 1 .

The relation-algebraic treatment of the certainty influence index is rather similar to that of the possibility influence index. However, we do not want to go into more details here, and we switch directly to followers.

The next theorem shows how sets of followers can be described relation-algebraically by means of column vectors in the sense of Subsection 3.2. In it, the relations $R$ and $Q$ column-wisely enumerate the sets $I_{S}$ and $B\left(I_{S}\right)$, respectively, and the column point $q$ again is used for specifying for $i \in I_{S}$ the specific Boolean value $i_{S}$. Once more it is arbitrarily which influence rule is used for the definition of the influence function $B$.

Theorem 4.3.3 Assume $s: P \leftrightarrow \mathbf{1}$ to describe the coalition $S \subseteq P$, and the column point $q \subseteq s$ to describe some player $k \in S$. Furthermore, let $\mathrm{M}: P \leftrightarrow 2^{P}$ be the membership relation. If the column vector follow $(D, s)$ of type $[P \leftrightarrow \mathbf{1}]$ is defined as

$$
\text { follow }(D, s):=\operatorname{syq}\left(Q^{\top}, R^{\top} q\right)
$$

with relations $R:=\mathrm{M} i n j\left(i s(s)^{\top}\right)^{\top}$ and $Q:=\operatorname{Dvec}(D) \operatorname{inj}\left(i s(s)^{\top}\right)^{\top}$, then for all $j \in P$ we have follow $(D, s)_{j, \perp}$ iff $j \in F_{B}(S)$.

Proof As in the proof of Theorem 4.3.2, we denote the subset of $2^{P}$ that is described by the row vector $i s(s): \mathbf{1} \leftrightarrow 2^{P}$ with $\mathcal{S}$. Then both $R$ and $Q$ have the type $[P \leftrightarrow \mathcal{S}]$. Furthermore, we are able to calculate as given below:

$$
\begin{aligned}
\text { follow }(D, s)_{j, \perp} & \Longleftrightarrow \operatorname{syq}\left(Q^{\top}, R^{\top} q\right)_{j, \perp} \\
& \Longleftrightarrow \forall X \in \mathcal{S}: Q_{X, j}^{\top} \leftrightarrow\left(R^{\top} q\right)_{X, \perp} \\
& \Longleftrightarrow \forall X \in \mathcal{S}: Q_{j, X} \leftrightarrow\left(q^{\top} R\right)_{\perp, X} \\
& \Longleftrightarrow \forall i \in I_{S}:(B i)_{j, \perp} \leftrightarrow i_{S}=1 \\
& \Longleftrightarrow j \in F_{S}(B)
\end{aligned}
$$

The fourth step of this calculation uses that there is a one-to-one corrrespondence between the sets $2^{P}$ and $I$, that $X \in 2^{P}$ belongs to $\mathcal{S}$ iff the corresponding inclination vector $i \in I$ belongs to $I_{S}$, that $\operatorname{Dvec}(D)$ column-wisely enumerates the decision vectors $B i$ and that $\left(q^{\top} R\right)_{\perp, X}$ iff $i_{S}=1$ (see the proof of Theorem 4.3.2).

Let us again demonstrate what the RELVIEW-program obtained from this theorem yields in the case of our running example with the coalition $S$ consisting of the players 2,3 and 5 .

Example 4.3.2. In the following two RELVIEW-pictures two column vectors are depicted which describe two subsets of the set $P$. The left column vector describes the set of followers of $S$ under the influence rule 'following only unambiguous trend-setters' and the right column vector does the same with 'following the majority of the trend-setters'. 

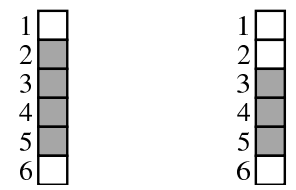

So, the followers of $S$ under the first rule are $2,3,4$ and 5 and those under the second rule are 3,4 and 5 .

Having a RELVIEW-program at hand for computing sets of followers, it is an easy task to implement another one that computes the kernel of an influence function by applying the former program to all subsets of $P$. Applied to our running example, the second program proved that there is no difference whether the influence function $B$ is defined via the rule 'following only unambiguous trend-setters' or the rule 'following the majority of the trend-setters'. Both rules yield the same result, viz. $\{\{6\},\{5\},\{2\},\{1\}\}$. Of course, this is a special case. Experiments with RELVIEW showed that, in general, the kernels of both rules we have introduced in this paper turn out to be different.

\section{The Dutch Parliament Example}

In the last section, we have used an artificial running example to illustrate our relation-algebraic approach to measure players' 'strength' in a social network. In the following we present another application of RELVIEw. It stems from the real world and is based on the structure of the Second Chamber (Tweede Kamer) of the present Dutch Parliament.

\subsection{The present Dutch parliament}

There are presently ten parties in the Dutch parliament, viz. (in alphabetic order) the parties CDA Christen-Democratisch Appel (Christian Democrats), CU - Christen Unie (Christian Union), D66 Democraten66 (Democrats 66), GL - GroenLinks (Green Left), PvdA - Partij van de Arbeid (Labor Party), PvdD - Partij voor de Dieren (Animal Party), PVV - Partij voor de Vrijheid (Party for Freedom), SGP - Staatkundig Gereformeerde Partij (Political Reformed Party), SP - Socialistische Partij (Socialist Party), and VVD - Volkspartij voor Vrijheid en Democratie (People's Party for Freedom and Democracy). Hence, we have

$$
P:=\{\mathrm{CDA}, \mathrm{CU}, \mathrm{D} 66, \mathrm{GL}, \mathrm{PvdA}, \mathrm{PvdD}, \mathrm{PVV}, \mathrm{SGP}, \mathrm{SP}, \mathrm{VVD}\} .
$$

In the following table the Dutch parties of the present parliament are shown $(k \in P)$, placed in a specific order, together with the numbers of seats $\left(w_{k}\right)$, where the total number of seats is equal to 150. The specific placement of the parties from GL to PVV is based on a left-right scale for the postwar period in the Netherlands, developed in [32] (see also [39]).

\begin{tabular}{c||c|c|c|c|c|c|c|c|c|c}
$k \in P$ & GL & SP & PvdA & D66 & PvdD & CDA & VVD & CU & SGP & PVV \\
\hline$w_{k}$ & 7 & 25 & 33 & 3 & 2 & 41 & 22 & 6 & 2 & 9
\end{tabular}

We like to mention two concepts developed within the framework of simple games, that can be applied to measure the 'strength' of political parties, i.e., the concepts of a dominant player ([34], see also $[15,17])$ and a central player $[16,17,19]$. For an empirical analysis of the effect of dominant and central parties on cabinets in Western multiparty democracies, see e.g., [37, 38, 39], and also [41]. A player $k$ is said to be a dominant player if there are coalitions $A, B$, such that $A \cap B=\emptyset$, $k \notin A \cup B, A \cup B$ is not a winning coalition, but $A \cup k$ and $B \cup k$ are winning coalitions. The dominant player is a 'policy blind' or 'office seeking' concept. In contrast to the dominant player, the concept of central player is a 'policy oriented' or 'policy seeking' one. Let players be ordered on 
a policy dimension. Player $k$ is said to be a central player if the connected coalition to the left of $k$ as well as the connected coalition to the right of $k$ can turn into a winning coalition only when $k$ joins this coalition.

Let us apply the concepts of dominant and central players to the present Dutch parliament. A winning coalition in this Dutch example is a coalition with at least 76 seats in the parliament. One may easily check that CDA is both the dominant and central player in the Dutch parliament. Indeed, none of the two disjoint coalitions PvdA-D66 and VVD-CU-PVV is winning, PvdA-D66-VVDCU-PVV is not winning either, but both PvdA-D66-CDA and VVD-CU-PVV-CDA are winning. Furthermore, both GL-SP-PvdA-D66-PvdD (i.e., the connected coalition to the left of CDA) and VVD-CU-SGP-PVV (i.e., the connected coalition to the right of CDA) can turn into a winning coalition when CDA joins.

The three parties CDA, CU and PvdA are presently forming the Dutch cabinet. We may assume that PvdA is a trend-setter for the two parties D66 and GL: the latter parties usually follow the former one. Furthermore, we assume that PVV is a trend-setter for VVD. This may be only partially true, but we assume it as hypothesis for our computations. Apart from that, we assume more influence relationships, which display both office seeking and policy seeking motivations of the Dutch parties. ${ }^{6}$ Let us assume that for some of the parties, the stronger (direct) neighbour on the left-right scale is a trend-setter of the party if this neighbour has more seats than the party in question. So, apart from being the trend-setter for D66, the party PvdA is assumed to be also the trend-setter for SP. The dominant and central party CDA is the trend-setter for VVD and PvdD (hence, VVD is assumed to have two trend-setters, PVV and CDA).

\subsection{Results of computations}

We have used the RELVIEW-versions of the relation-algebraic specifications of Section 4 to determine by means of the tool for this quasi-realistic example all the concepts mentioned in Section 2. Since each party has at most two trend-setters, both influence rules of Subsection 2.3 lead to the same description of the influence function $B$. We have for each inclination vector $i$ that $(B i)_{\mathrm{D} 66}=(B i)_{\mathrm{SP}}=(B i)_{\mathrm{GL}}=i_{\mathrm{PvdA}},(B i)_{\mathrm{PvdD}}=i_{\mathrm{CDA}},(B i)_{\mathrm{VvD}}$ depends on the inclinations $i_{\mathrm{CDA}}$ and $i_{\mathrm{PVV}}$ and $(B i)_{j}=i_{j}$ for $j$ being PvdA, CDA, CU, SGP, PVV. Please note that with the influence relationships we adopt in this example, no party in the Dutch cabinet has a trend-setter. As example for a coalition $S$ we have taken the three parties which form the present Dutch cabinet, i.e., $S:=\{\mathrm{CDA}, \mathrm{CU}, \mathrm{PvdA}\}$. Here is the RELVIEW-representation of the dependency relation and the coalition $S$.

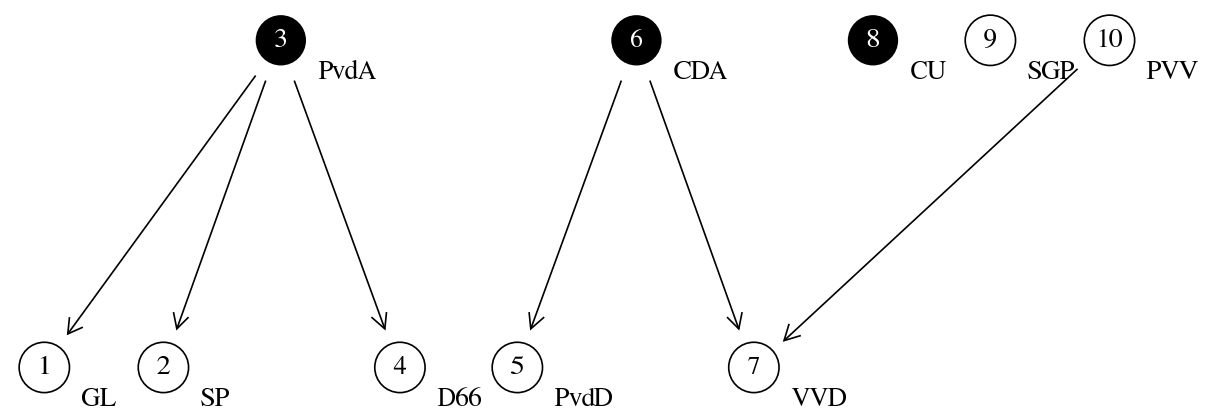

There are situations where parties decide without taking their numbers of seats into account, for instance, in parliamentary committees with one representative per party. In such situations the group decision $g d$ is given by simple majority of the number of parties. For each Dutch party

\footnotetext{
${ }^{6}$ We have also investigated the Dutch parliament example only with the trend-setter relationships PvdA-D66 and PvdA-GL. But we think that the inclusion of more relationships makes it more interesting.
} 
$k \in P$, we determined the Hoede-Bakker index $\operatorname{HB}_{k}(B, g d)$, the generalized Hoede-Bakker index $\mathrm{GHB}_{k}(B, g d)$ and its modifications. Moreover, for each party outside the cabinet, that is, for all $j \in P \backslash S$, we have calculated the possibility and certainty influence indices of the cabinet on $j$, i.e., $\bar{d}(B, S \rightarrow j)$ and $\underline{d}(B, S \rightarrow j)$, as well as the set of followers of the cabinet $F_{B}(S)$ under $B$, and the kernel $\mathcal{K}(B)$ of $B$. Here are some results. Because of their sizes we are not able to present the corresponding RELVIEW-matrices. We only present the decisive numbers that, as already mentioned, either are directly delivered by RELVIEW as numbers of 1-entries of computed results or can be easily computed from these numbers.

Let us start with the power indices. In the following table we listen for the Dutch parliament example the sizes of the sets underlying their definitions as computed by RELVIEw using the relation-algebraic specifications of the Subsections 4.1 and 4.2.

\begin{tabular}{c||c|c|c|c|c|c|c|c|c|c}
$k \in P$ & GL & SP & PvdA & D66 & PvdD & CDA & VVD & CU & SGP & PVV \\
\hline$\left|I_{k}^{++}\right|$ & 216 & 216 & 400 & 216 & 216 & 288 & 224 & 256 & 256 & 272 \\
$\left|I_{k}^{+-}\right|$ & 296 & 296 & 112 & 296 & 296 & 224 & 288 & 256 & 256 & 240 \\
$\left|I_{k}^{-+}\right|$ & 216 & 216 & 32 & 216 & 216 & 144 & 208 & 176 & 176 & 160 \\
$\left|I_{k}^{--}\right|$ & 296 & 296 & 480 & 296 & 296 & 368 & 304 & 336 & 336 & 352
\end{tabular}

From these numbers and the fact that $\left|I^{+}\right|=432$ and $\left|I^{-}\right|=1024-432=592$, we immediately are able to compute all power indices introduced in Subsection 2.1. In the following table we show the values for the two power indices $\mathrm{HB}_{k}$ and $\mathrm{GHB}_{k}$ only.

\begin{tabular}{c||c|c|c|c|c|c|c|c|c|c}
$k \in P$ & GL & SP & PvdA & D66 & PvdD & CDA & VVD & CU & SGP & PVV \\
\hline $\mathrm{HB}_{k}$ & -0.16 & -0.16 & 0.56 & -0.16 & -0.16 & 0.12 & -0.12 & 0 & 0 & 0.06 \\
$\mathrm{GHB}_{k}$ & 0 & 0 & 0.72 & 0 & 0 & 0.28 & 0.03 & 0.16 & 0.16 & 0.22
\end{tabular}

In their paper Hoede and Bakker have proven that if, first, changing all inclinations leads to a change of the group decisions and, second, the decision function $g d$ is monotone, then the value of $\mathrm{HB}_{k}(B, g d)$ belongs to the interval $[0,1]$ of the real numbers. The reason that we obtain negative values for the Hoede-Bakker index is that the first axiom of [25] does not hold for the Dutch parliament example. With the help of RELVIEW we obtained that from the possible 1024 inclination vectors exactly 160 inclination vectors $i$ do not fulfil the equation $g d(B(\bar{i}))=\neg g d(B i)$. An example is the following one:

$$
i_{\mathrm{GL}}=i_{\mathrm{SP}}=i_{\mathrm{PvdA}}=i_{\mathrm{D} 66}=i_{\mathrm{PvdD}}=i_{\mathrm{VVD}}=i_{\mathrm{CU}}=0 \quad i_{\mathrm{CDA}}=i_{\mathrm{SGP}}=i_{\mathrm{PVV}}=1
$$

Here the influences given by the above graph only change the inclinations 'no' of the parties PvdD and VVD to the decision 'yes', the rest remains unchanged. With five 'yes' decisions the group decision is 'no'. In the case of the complement of $i$ we get now for exactly the same parties a change of the inclinations 'yes' to the decisions 'no' and again the rest remains unchanged. This also leads to 'no' as group decision.

The reason for the introduction of the generalized Hoede-Bakker index in [42] was to avoid the first axiom of Hoede and Bakker. It is not satisfied if there is a vetoer in the social network (as already mentioned in Subsection 2.1) and also causes serious problems with an even number of players in the social network, as in our Dutch parliament example. If five of the Dutch parties vote 'yes' and the remaining five parties vote 'no', then the axiom does not hold. Instead of the first axiom of Hoede and Bakker for the generalized Hoede-Bakker index it suffices to demand that the inclination vector $(1, \ldots, 1)$ (in terms of relation algebra: the universal column vector $L$ ) leads to the group decision 'yes' and the inclination vector $(0, \ldots, 0)$ (the empty column vector $\mathrm{O}$, respectively) leads to the group decision 'no'.

Since the specification of the row vector $g d v(D): \mathbf{1} \leftrightarrow 2^{P}$ of Theorem 4.1.2 bases on 'simple majority of number of parties' as group decision function $g d$, in our concrete example meaning that 
$g d(B i)=1$ iff the size of the set $\left\{j \in P \mid(B i)_{j}=1\right\}$ is at least 6 , in the above results all parties are treated as if they have exactly one seat. However, in plenary meetings of the Dutch parliament the number of seats is decisive. Here a proposal is accepted by the parliament iff more than 75 seats vote 'yes'. ${ }^{7}$ As a consequence, the majority-of-parties-based definition of $g d$ we have used so far may lead to wrong results, if, e.g., 5 parties with few seats vote 'no' while the proposal is accepted because more than 75 seats vote 'yes'. An example for this is:

$$
i_{\mathrm{CDA}}=i_{\mathrm{SGP}}=i_{\mathrm{PVV}}=0 \quad i_{j}=1 \text { for } j \notin\{\mathrm{CDA}, \mathrm{SGP}, \mathrm{PVV}\}
$$

The influences given by the above graph changes the inclinations 'yes' of the two parties PvdD and VVD to the decision 'no', the remaining parties vote according to their inclinations. With five 'no' and five 'yes' the majority-of-parties-based definition of $g d$ leads to group decision 'no'. But the parliament votes 'yes' due to the $2+41+22+2+9=76$ seats of the five parties PvdD, CDA, VVD, SGP and PVV together.

If we assume that each party votes as a block, then the following alternative definition of the group decision function precisely describes how the Dutch parliament makes a decision:

$$
g d(B i)=1 \Longleftrightarrow \sum_{k \in P_{i}^{+}} w_{k}>75
$$

where $P_{i}^{+}:=\left\{j \in P \mid(B i)_{j}=1\right\}$ is the set of parties which vote 'yes' under inclination vector $i$ and $w_{k}$ denotes the number of seats of party $k \in P$ according to the table of Subsection 5.1.

To obtain a relation-algebraic specification of the row vector $g d v(D): 1 \leftrightarrow 2^{P}$ also for the majority-of-seats-based group decision function introduced by (16), we assume $N$ to be the set of the 150 Dutch parliament seats and the distribution of the seats over the parties to be given by a relation $W: N \leftrightarrow P$ such that

$$
W_{n, k} \Longleftrightarrow n \text { is ownded by } k
$$

for all $n \in N$ and $k \in P$. The relation $W$ is a mapping in the relational sense and for each $k \in P$ the $k$-column of $W$ consists of $w_{k} 1$-entries and $150-w_{k} 0$-entries. Now, let $i: P \leftrightarrow \mathbf{1}$ be an arbitrary inclination vector and $B i: P \leftrightarrow \mathbf{1}$ the corresponding decision vector (as relationalgebraically specified in Theorem 4.1.1). Then we have for all $n \in N$ the equivalence

$$
(W B i)_{n, \perp} \Longleftrightarrow \exists k \in P: W_{n, k} \wedge(B i)_{k, \perp} \Longleftrightarrow \exists k \in P_{i}^{+}: W_{n, k}
$$

such that the column vector $W B i: N \leftrightarrow \mathbf{1}$ describes the set of seats $N_{i}^{+} \in 2^{N}$ which are owned by a party that votes 'yes' under inclination vector $i$. Since the relation-algebraic expression that specifies the column vector $B i$ is built from $i$ using unions, intersections, complements and leftcompositions with constants only, the same holds for the expression $W B i$. Hence, a replacement of $B i$ in the latter by the column-wise enumeration of all decision vectors, i.e., by the relation Dvec $(D): P \leftrightarrow 2^{P}$ of (13), leads to the column-wise enumeration of all sets $N_{i}^{+}$. With respect to the row vector $g d v(D)$ we are looking for, this means that for the relation $W \operatorname{Dvec}(D): N \leftrightarrow 2^{P}$ and for all sets $X \in 2^{P}$ the following property has to hold: If the inclination vector $i: P \leftrightarrow \mathbf{1}$ equals the $X$-column of the membership relation $\mathrm{M}: P \leftrightarrow 2^{P}$, then

$$
g d v(D)_{\perp, X} \Longleftrightarrow \text { the } X \text {-column of } W \operatorname{Dvec}(D) \text { contains at least } 76 \text { 1-entries. }
$$

From (17) a relation-algebraic specification of $g d v(D)$ can be obtained exactly as in the case of Theorem 4.1.2 for the majority-of-parties-based group decision function, i.e., by using a threshold vector, the operation cardfilter and a symmetric quotient construction. Here is the corresponding

\footnotetext{
${ }^{7}$ Of course, this only holds if each member attends the House.
} 
RELVIEW-code, where, compared with the program of Subsection 4.1, $W$ is an additional argument and again the argument $w$ determines the threshold for majority of seats by its length, now 76 .

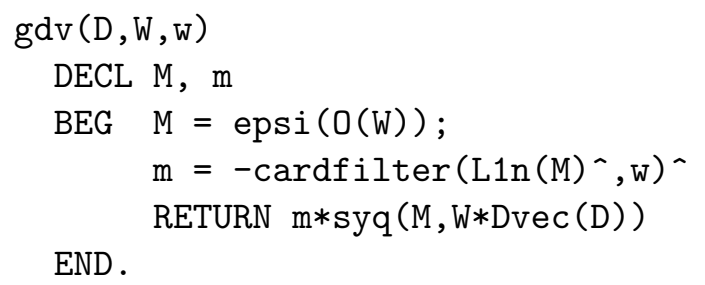

If we use this version of $g d v$ instead of the version of Subsection 4.1, then we obtain the following sizes of the sets underlying the definitions of the various power indices.

\begin{tabular}{c||c|c|c|c|c|c|c|c|c|c}
$k \in P$ & GL & SP & PvdA & D66 & PvdD & CDA & VVD & CU & SGP & PVV \\
\hline$\left|I_{k}^{++}\right|$ & 256 & 256 & 416 & 256 & 256 & 352 & 256 & 288 & 288 & 352 \\
$\left|I_{k}^{+-}\right|$ & 256 & 256 & 96 & 256 & 256 & 160 & 256 & 224 & 224 & 160 \\
$\left|I_{k}^{-+}\right|$ & 256 & 256 & 96 & 256 & 256 & 160 & 256 & 224 & 224 & 160 \\
$\left|I_{k}^{--}\right|$ & 256 & 256 & 416 & 256 & 256 & 352 & 256 & 288 & 288 & 352
\end{tabular}

Since for all $k \in P$ we have $\left|I_{k}^{++}\right|=\left|I_{k}^{--}\right|$and $\left|I_{k}^{+-}\right|=\left|I_{k}^{-+}\right|$, now the values $\mathrm{HB}_{k}$ and $\mathrm{GHB}_{k}$ coincide. In this new case $\left|I^{+}\right|=\left|I^{-}\right|=512$ and thus from the values of the table above also all other power indices of Subsection 2.1 can be obtained. In the following table we show the values for the power index $\mathrm{GHB}_{k}$.

\begin{tabular}{c||c|c|c|c|c|c|c|c|c|c}
$k \in P$ & GL & SP & PvdA & D66 & PvdD & CDA & VVD & CU & SGP & PVV \\
\hline $\mathrm{GHB}_{k}$ & 0 & 0 & 0.62 & 0 & 0 & 0.37 & 0 & 0.12 & 0,12 & 0.37
\end{tabular}

Comparing this table with the table presenting $\mathrm{GHB}_{k}$ for the previous case, we see that in case of the two largest parties PvdA (33 seats) and CDA (41 seats), the net Success of PvdA (measured by $\left.\mathrm{GHB}_{\mathrm{PvdA}}\right)$ increases but the net Success of CDA decreases if decisions are transfered from the plenum to parliamentary commissions.

We have used the above RELVIEW-program $g d v$ and the RELVIEW-program $g d v$ of Subsection 4.1 also to find all inclination vectors for which majority-of-seats-based decision differs from majority-of-parties-based decision. RELVIEW delivered the following result: If the majority-ofparties-based decision is 'yes', then the same holds for the majority-of-seats-based decision. The other direction does not hold. There are exactly 80 inclination vectors which lead to 'yes' if the decision bases on the majority of seats and to 'no' if it bases on the majority of parties. In each of these cases the inclination of PVV is 'yes'.

Having discussed power indices in great detail, let us now present the RELVIEW results concerning the two influence indices, sets of followers and kernels. Note that for computing these values the group decision function $g d$ does not play a role.

The next table shows for all Dutch parties not being part of the coalition $S=\{\mathrm{CDA}, \mathrm{CU}, \mathrm{PvdA}\}$ (the present Dutch cabinet) the sizes of the sets defining the possibility influence indices $\bar{d}(B, S \rightarrow j)$ as computed by RELVIEW.

\begin{tabular}{c||c|c|c|c|c|c|c}
$j \notin S$ & GL & SP & D66 & PvdD & VVD & SGP & PVV \\
\hline$\left|I_{S \rightarrow j}\right|$ & 128 & 128 & 128 & 128 & 128 & 128 & 128 \\
$\left|I_{S \rightarrow j}^{*}\right|$ & 128 & 128 & 128 & 128 & 64 & 0 & 0
\end{tabular}

Hence, the possibility influence index of $S$ on GL, SP, D66 and PvdD is 1 (denoting maximal influence), the possibility influence index of $S$ on VVD is 0.5 and the possibility influence index of $S$ on SGP and PVV is 0 (denoting no influence). 
The results for the certainty influence indices of the present cabinet on the remaining parties are as follows. For $j$ being GL, SP, D66 or PvdD, from the 128 inclination vectors of $I_{S \rightarrow j}^{*}$ exactly two show for all parties outside $S$ an inclination that is different from the inclination of $S$. Hence, in these cases the certainty influence index is 1 . None of the 64 inclination vectors of $I_{S \rightarrow \mathrm{VVD}}^{*}$ has this property, so the certainty influence index of $S$ on VVD is 0 . That the certainty influence index of $S$ on the remaining parties SGP and PVV is 0 follows from $\left|I_{S \rightarrow j}^{*}\right|=0$ for $j \in\{\mathrm{SGP}, \mathrm{PVV}\}$.

Let us, finally, present the results concerning the set of followers and kernels. We obtained with the help of the RELVIEW tool that

\section{$\{\mathrm{GL}, \mathrm{SP}, \mathrm{PvdA}, \mathrm{D} 66, \mathrm{PvdD}, \mathrm{CDA}, \mathrm{CU}\}$}

is the set of the seven followers of the present cabinet $S$, and that the set of singleton sets

$$
\{\{\mathrm{PvdA}\},\{\mathrm{CDA}\},\{\mathrm{CU}\},\{\mathrm{SGP}\},\{\mathrm{PVV}\}\}
$$

is the kernel of the influence function induced by the above dependency graph and both influence rules we have considered in Subsection 2.3.

\section{Conclusions}

The present paper disseminates part of our results on applications of relation algebra and the Kiel ReLVIEw tool to Game Theory and Social Choice Theory. In our previous works $[7,8]$, we presented such applications to coalition formation, where with the help of relation algebra and RELVIEW the set of all feasible stable governments has been determined. In the present paper, we apply relation algebra and RELVIEW to network formation, i.e., to compute some measures of players' strength, like power, success, and influence, in a network.

What we particularly like in this approach is its usefulness with respect to applying the tools to organizations and trend-setter structures with a larger number of players. One of the straightforward ideas is to apply the measures calculated by RELVIEW to parliaments. This is what we presented in the previous section, where an application of the tools in question to the Dutch parliament is delivered. For networks with more than, say 5 or 6 players, where some of the players have trend-setters that they follow, calculating the measures and concepts of influence, like the HoedeBakker index, its generalization and modifications, the set of followers and the kernel of an influence function, is far too complicated to be done by hand due to the sizes of the sets $I$ of inclinations. In case of networks with, say, 25 players, even a naive Boolean vector approach within a conventional programming language like $\mathrm{C}$ or Java leads to serious difficulties. It is hardly to imagine to generate all the $2^{25}=33.554 .432$ Boolean column vectors of length 25 one after another and to transform them into the corresponding decision vectors within reasonable time. Due to the very efficient BDDimplementation of relations, the RELVIEw tool allows to do this in a very efficient and elegant way in many cases, viz. if programs essentially are described by relation-algebraic expressions and do not use loops that range over huge sets. To give an impression of the amazing power of the BDD-implementation of relations, we want to mention that RELVIEW needs on a Sun Fire-280R workstation $(750 \mathrm{MHz}, 4$ GByte main memory, running Solaris) only 0.04 seconds to compute the group decision vector using the program $g d v$ of Subsection 5.2. Note that the symmetric quotient $\operatorname{syq}(\mathrm{M}, W D \operatorname{vec}(D))$ used in $g d v$ has type $\left[2^{N} \leftrightarrow 2^{N}\right]$. Regarded as a Boolean matrix, this means that it has $2^{150}$ rows and columns.

Of course, we also are not able to compute within reasonable time with RELVIEW the kernel of an influence function in the case of, say again, 25 players, if we apply the program for computing sets of followers to all subsets of $P$, i.e., all possible column vectors $\mathrm{M} p: P \leftrightarrow \mathbf{1}$ with $p: \mathbf{1} \leftrightarrow 2^{P}$ being a row point, via a simple loop. A strategy that may help in such a situation is to estimate the 
sizes of the sets of the kernel. If we are able to show by an analysis of the dependency relationships, for instance, that each set of the kernel consists of at most 3 players, then we can use the baseoperation cardfilter to obtain a row vector $v: \mathbf{1} \leftrightarrow 2^{P}$ that represents the set $\left\{X \in 2^{P}|| X \mid \leq 3\right\}$. In the case of 25 players, instead of all 33.554 .432 column vectors $\mathrm{M} p: P \leftrightarrow \mathbf{1}$ we then only have to loop through the $\sum_{m=0}^{3}\left(\begin{array}{c}25 \\ m\end{array}\right)=14.426$ possible row vectors $\mathrm{M} p$ with the row point $p$ contained in $v$.

A further advantage of the approach of the present paper is that, because of the concise form of the RELVIEW-programs and the expressiveness of the relation-algebraic language, the combination of both tools additionally allows to experiment with given concepts and concepts being still under development without having a large overhead and, furthermore, to animate computations and to visualize their results. There are many more possibilities to combine relation algebra and RELVIEW to investigate and solve problems from Game Theory and Social Choice Theory. One of them could be an application of the tools in question to bargaining theory, in particular, to determining solutions of bargaining games.

Acknowledgement. We like to thank Adrian Van Deemen for his suggestions concerning the example based on the Dutch Parliament and Stefan Bolus for his further development of RELVIEW.

\section{References}

[1] J. Banzhaf, Weighted voting doesn't work: a mathematical analysis, Rutgers Law Review 19, 317-343 (1965)

[2] R. Behnke, R. Berghammer, E. Meyer and P. Schneider, RelView - A system for calculation with relations and relational programming, In: E. Astesiano (ed.), Proc. Conf. "Fundamental Approaches to Software Engineering (FASE '98)", LNCS 1382, Springer, 318-321 (1998)

[3] R. Berghammer and T. Hoffmann, Modelling sequences within the RELVIEW system, Journal of Universal Computer Science 7, 107-123 (2001)

[4] R. Berghammer, B. Karger von and C. Ulke, Relation-algebraic analysis of Petri nets with RelView, In: T. Margaria and B. Steffen (eds.), Proc. 2nd Workshop "Tools and Applications for the Construction and Analysis of Systems (TACAS '96)", LNCS 1055, Springer, 49-69 (1996)

[5] R. Berghammer, B. Leoniuk and U. Milanese, Implementation of relational algebra using binary decision diagrams, In: H. Swart de (ed.), Proc. 6th Int. Workshop "Relational Methods in Computer Science", LNCS 2561, Springer, 241-257 (2002)

[6] R. Berghammer, Relation-algebraic computation of fixed points with applications, Journal of Logic and Algebraic Programming, 66, 112-126 (2006)

[7] R. Berghammer, A. Rusinowska and H. Swart de, Applying relational algebra and RelView to coalition formation, European Journal of Operational Research 178/2, 530-542 (2007)

[8] R. Berghammer, A. Rusinowska and H. Swart de, An interdisciplinary approach to coalition formation, European Journal of Operational Research 195, 487-496 (2009)

[9] R. Berghammer, G. Schmidt and M. Winter, RELVIEW and RATH - Two systems for dealing with relations, In: [49], 1-16 (2003)

[10] S.J. Brams, W.F. Lucas and P.D. Straffin, Political and Related Models, Volume 2 in series Models in Applied Mathematics, New York: Springer (1982)

[11] C. Brink, W. Kahl and G. Schmidt (eds.), Relational Methods in Computer Science, Advances in Computing Science, Springer (1997)

[12] J.S. Coleman, Control of collectivities and the power of a collectivity to act, In: Lieberman, Gordon and Breach Social Choice, London (1971)

[13] J.S. Coleman, Individual Interests and Collective Action: Selected Essays, Cambridge University Press (1986) 
[14] J. Deegan and E.W. Packel, A new index of power for simple n-person games, International Journal of Game Theory 7, 113-123 (1978)

[15] A. Deemen van, Dominant players and minimum size coalitions, European Journal of Political Research 17, 313-332 (1989)

[16] A. Deemen van, Coalition formation in centralized policy games, Journal of Theoretical Politics 3, 139-161 (1991)

[17] A. Deemen van, Coalition Formation and Social Choice, Boston: Kluwer Academic Publishers (1997)

[18] P. Dubey and L.S. Shapley, Mathematical properties of the Banzhaf power index, Mathematics of Operations Research 4, 99-131 (1979)

[19] E. Einy, On connected coalitions in dominated simple games, International Journal of Game Theory 14, 103-125 (1985)

[20] D.S. Felsenthal and M. Machover, The Measurement of Voting Power: Theory and Practice, Problems and Paradoxes, London: Edward Elgar Publishers (1998)

[21] M. Grabisch and A. Rusinowska, Measuring influence among players with an ordered set of possible actions GATE Working Paper, 2008-01, GATE, Ecully, France (2008)

[22] M. Grabisch and A. Rusinowska, Influence functions, followers and command games, Submitted for publication (2008)

[23] M. Grabisch and A. Rusinowska, A model of influence in a social network, Theory and Decision, forthcoming (2009)

[24] M. Grabisch and A. Rusinowska, Measuring influence in command games, Social Choice and Welfare, forthcoming (2009)

[25] C. Hoede and R. Bakker, A theory of decisional power, Journal of Mathematical Sociology 8, 309-322 (1982)

[26] M.J. Holler, Forming coalitions and measuring voting power, Political Studies 30, 262-271 (1982)

[27] M.J. Holler and E.W. Packel, Power, luck and the right index, Journal of Economics 43, 21-29 (1983)

[28] R.J. Johnston, On the measurement of power: Some reactions to Laver, Environment and Planning A 10, 907-914 (1978)

[29] T. König and T. Bräuninger, The inclusiveness of European decision rules, Journal of Theoretical Politics 10, 125-142 (1998)

[30] A. Laruelle and F. Valenciano, Assessing success and decisiveness in voting situations, Social Choice and Welfare 24, 171-197 (2005)

[31] W.F. Lucas, Measuring power in weighted voting games, In: [10], 183-238 (1982)

[32] M.J. Morgan, The Modelling of governmental coalition formation: A policy-based approach with interval measurement, Doctoral Dissertation, University of Michigan (1976)

[33] G. Owen, Game Theory, San Diego: Academic Press (1995)

[34] B. Peleg, Coalition formation in simple games with dominant players, International Journal of Game Theory 10, 11-33 (1981)

[35] L.S. Penrose, The elementary statistics of majority voting, Journal of the Royal Statistical Society 109, 53-57 (1946)

[36] D. Rae, Decision rules and individual values in constitutional choice, American Political Science Review 63, 40-56 (1969)

[37] P. Roozendaal van, The effect of dominant and central parties on cabinet composition and durability, Legislative Studies Quarterly 17, 5-36 (1992)

[38] P. Roozendaal van, Cabinets in Multi-Party Democracies, Amsterdam: Thesis Publishers (1992)

[39] P. Roozendaal van, Cabinets in the Netherlands (1918-1990): The importance of 'dominant' and 'central' parties, European Journal of Political Research 23, 35-54 (1993) 
[40] A. Rusinowska, On the not-preference-based Hoede-Bakker index, Forthcoming in: L. Petrosjan and V. Mazalov (eds.) Game Theory and Applications, Vol. XIII, Nova Science Publishers (2008)

[41] A. Rusinowska, The Hoede-Bakker index modified to the Shapley-Shubik and Holler-Packel indices, Submitted for publication (2008)

[42] A. Rusinowska and H. Swart de, Generalizing and modifying the Hoede-Bakker index, In: [50], 60-88 (2006)

[43] A. Rusinowska and H. Swart de, On some properties of the Hoede-Bakker index, Journal of Mathematical Sociology 31(4), 267-293 (2007)

[44] A. Rusinowska, H. Swart de and J.-W. Rijt van der, A new model of coalition formation, Social Choice and Welfare 24, 129-154 (2005)

[45] A. Rusinowska, R. Berghammer, P. Eklund, J.-W. Rijt van der, M. Roubens and H. Swart de, Social software for coalition formation, In: [50], 1-30 (2006)

[46] L.S. Shapley and M. Shubik, A method for evaluating the distribution of power in a committee system, American Political Science Review 48, 787-792 (1954)

[47] G. Schmidt and T. Ströhlein, Relations and Graphs, Discrete Mathematics for Computer Scientists, EATCS Monographs on Theoret. Comput. Sci., Springer (1993)

[48] P.D. Straffin, Power indices in politics, In: [10], 256-321 (1982)

[49] H. Swart de, E. Orlowska, G. Schmidt and M. Roubens (eds.), Theory and Applications of Relational Structures as Knowledge Instruments, LNCS 2929, Springer (2003)

[50] H. Swart de, E. Orlowska, G. Schmidt and M. Roubens (eds.), Theory and Applications of Relational Structures as Knowledge Instruments II, LNAI 4342, Springer (2006) 